\title{
A Preorganized Electric Field Leads to Minimal Geometrical Reorientation in the Catalytic Reaction of Ketosteroid Isomerase
}

\author{
Yufan Wu, Stephen Fried, Steven Boxer
}

Submitted date: 09/01/2020 - Posted date: 10/01/2020

Licence: CC BY-NC-ND 4.0

Citation information: Wu, Yufan; Fried, Stephen; Boxer, Steven (2020): A Preorganized Electric Field Leads to Minimal Geometrical Reorientation in the Catalytic Reaction of Ketosteroid Isomerase. ChemRxiv. Preprint. https://doi.org/10.26434/chemrxiv.11559660.v1

Electrostatic interactions play a pivotal role in enzymatic catalysis and are increasingly modeled explicitly in computational enzyme design; nevertheless, they are challenging to measure experimentally. Using vibrational Stark effect (VSE) spectroscopy, we have measured electric fields inside the active site of the enzyme ketosteroid isomerase (KSI). These studies have shown that these fields can be unusually large, but it has been unclear to what extent they specifically stabilize the transition state (TS) relative to a ground state (GS). In the following, we use crystallography and computational modeling to show that KSI's intrinsic electric field is nearly perfectly oriented to stabilize the geometry of its reaction's TS. Moreover, we find that this electric field adjusts the orientation of its substrate in the ground state so that the substrate needs to only undergo minimal structural changes upon activation to its TS. This work provides evidence that the active site electric field in $\mathrm{KSI}$ is preorganized to facilitate catalysis and provides a template for how electrostatic preorganization can be measured in enzymatic systems.

File list (2)

Preorganization final_YW.pdf (660.36 KiB)

view on ChemRxiv - download file 


\title{
A Preorganized Electric Field Leads to Minimal Geometrical Reorien- tation in the Catalytic Reaction of Ketosteroid Isomerase
}

\author{
Yufan $\mathrm{Wu}^{\dagger}$, Stephen D. Fried ${ }^{\ddagger}$ and Steven G. Boxer* \\ Department of Chemistry, Stanford University, Stanford, California 94305-5012, United States
}

\begin{abstract}
Electrostatic interactions play a pivotal role in enzymatic catalysis and are increasingly modeled explicitly in computational enzyme design; nevertheless, they are challenging to measure experimentally. Using vibrational Stark effect (VSE) spectroscopy, we have measured electric fields inside the active site of the enzyme ketosteroid isomerase (KSI).1,2 These studies have shown that these fields can be unusually large, but it has been unclear to what extent they specifically stabilize the transition state (TS) relative to a ground state (GS). In the following, we use crystallography and computational modeling to show that KSI's intrinsic electric field is nearly perfectly oriented to stabilize the geometry of its reaction's TS. Moreover, we find that this electric field adjusts the orientation of its substrate in the ground state so that the substrate needs to only undergo minimal structural changes upon activation to its TS. This work provides evidence that the active site electric field in KSI is preorganized to facilitate catalysis and provides a template for how electrostatic preorganization can be measured in enzymatic systems.
\end{abstract}

Electrostatic stabilization has been widely discussed as an important feature that endows enzymes with their signature high catalytic proficiencies. ${ }^{3-9}$ One (simple) way to frame this hypothesis is that enzymes create a particular electrostatic environment in their active sites, which preferentially stabilizes the charge distribution of the transition state (TS) more than the ground state (GS) to accelerate the reaction 4 :

$$
\Delta \Delta G^{\ddagger}=-\left(\left(\vec{F}_{\text {enz,TS }} \cdot \vec{\mu}_{\mathrm{TS}}\right)-\left(\vec{F}_{\mathrm{enz}, \mathrm{R}} \cdot \vec{\mu}_{\mathrm{R}}\right)\right)
$$

where $\vec{\mu}_{\mathrm{R}}$ is the reactant's dipole moment, $\vec{\mu}_{\mathrm{TS}}$ is the transition state's dipole moment, $\vec{F}_{\text {enz,R }}$ is the electric field the environment exerts on the reactant dipole, and $\vec{F}_{\text {enz,Ts }}$ is the electric field the environment exerts on the transition state dipole. It should be noted that Eq. 1 exactly treats the simple case where the substrate is a point-dipole; in more realistic scenarios, $\Delta \Delta G^{\ddagger}$ is a sum of several terms for each bond dipole that changes during the reaction coordinate, in which the fields correspond to the fields projected onto the given bond dipoles at their respective positions.

Conceptually, electrostatic stabilization can be divided into two limiting cases. ${ }^{6}$ In one case, where the dipole moment of the substrate does not reorient upon activation, preferential stabilization of the TS can be achieved because the magnitude of the dipole moment in the TS is larger than that in the GS, which here we call a scaling effect. In a second case, where the dipole moment of the substrate reorients but does not change its magnitude upon activation, preferential stabilization can be achieved because the orientation of the dipole moment in the TS is better aligned with the electric field of the enzyme, which we call an orientational effect.

A

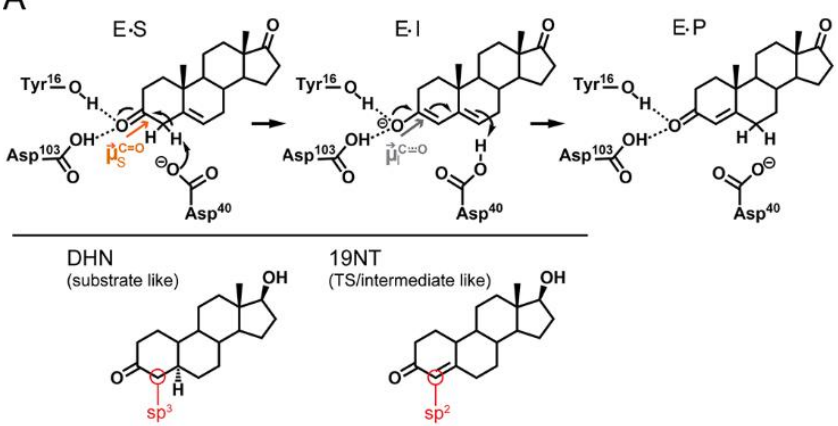

B

substrate (5-androstene)
transition state transition state
intermediate

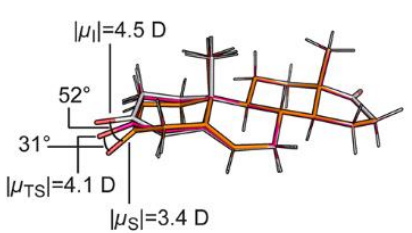

C

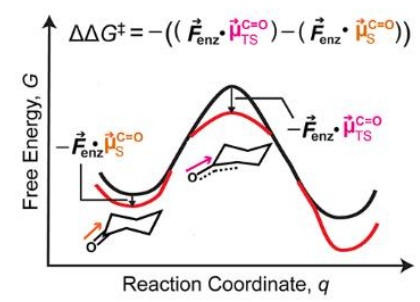

Figure 1. Electrostatic stabilization in the context of ketosteroid isomerase (KSI). (A) KSI catalyzes the isomerization of 5 androstenedione to 4 -androtenedione via a dienolate intermediate. The negative charge on the oxygen in the transition state (TS) and intermediate state (I) is stabilized by the oxyanion hole. As the reaction proceeds from the GS to the TS and I, the hybridization of the $\mathrm{C} 4$ carbon (red circle) of the substrate changes from $\mathrm{sp}^{3}$ to $\mathrm{sp}^{2}$. $5 \alpha$-dihydronandrolone (DHN) and 19nortestosterone (19NT) are used to mimic the two states respectively. (B) Ab initio calculations on the substrate, TS, and I of KSI's reaction in the gas phase suggests electrostatic and geometric changes occur along the reaction coordinate. (C) The electric field of KSI, $\vec{F}_{\text {enz, }}$ preferentially stabilizes the TS because of its larger bond dipole. Furthermore, the angle change shown in (B) could result in the $\mathrm{C}-\mathrm{O}$ dipole in the TS becoming better

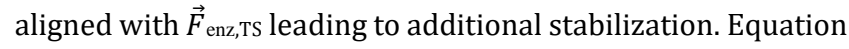


1 encompasses both the scaling effect and orientational effect of electrostatic catalysis.

Previous work in our lab has demonstrated a significant role of electrostatic stabilization in the catalytic proficiency of the model enzyme ketosteroid isomerase (KSI)..$^{1,2} \mathrm{KSI}$ catalyzes the isomerization of 5-androstenedione to 4-androstenedione via a dienolate intermediate with a reaction rate approximately 1011.5-fold faster than that of the 'uncatalyzed' reaction that proceeds through the same nominal mechanism. ${ }^{3,10}$ An oxyanion hole composed of tyrosine 16 (Tyr ${ }^{16}$ ) and aspartic acid 103 (Asp $\left.{ }^{103}\right)$ in the active site directly interacts with the carbonyl group of the substrate that undergoes a charge rearrangement in KSI's rate limiting step (Fig. $1 \mathrm{~A}$ lower). Tyr ${ }^{16}$ is further hydrogen-bonded (H-bonded) with tyrosine 57 and tyrosine 32 to form an extended H-bond network (Fig. S1A). ${ }^{11}$ Using a combination of vibrational Stark effect (VSE) spectroscopy, solvatochromic data and molecular dynamic (MD) simulations, the electric field experienced by the carbonyl group of a TS/I ana$\log$, 19-nortestosterone (19NT), was found to be very large when bound in the KSI active site. Moreover, this active site electric field (as probed by the carbonyl group of 19NT) is linearly correlated with the free energy barrier of the catalyzed reaction, $\Delta G^{\ddagger}$, across wild-type KSI and several KSI mutants with canonical and noncanonical amino acid substitutions (Fig. S1B). ${ }^{1,2}$

In previous work modeling the role of electrostatics in catalysis KSI, we employed the simplifying assumption that the orientation of the substrate's $\mathrm{C}=0$ does not change significantly during KSI's catalysis and that the (constant) $\mathrm{C}=0$ orientation could be modeled with 19NT. In this model, Eq. 1 simplifies to the following expression:

$$
\Delta \Delta G^{\ddagger}=-\left|\vec{F}_{\text {enz }}\right|\left(\left|\vec{\mu}_{\mathrm{TS}}\right|-\left|\vec{\mu}_{\mathrm{R}}\right|\right)
$$

from which one would expect to see a linear correlation between activation barrier and active site electric field. This model assumes that reorientation of the substrate's $\mathrm{C}=0$ dipole upon formation of the TS is minimal and electrostatic stabilization in KSI works entirely through the scaling effect. Under this assumption, VSE measurements showed that the carbonyl bond of the substrate increases in magnitude by $1.1 \mathrm{D}$ upon passage to the TS and electrostatic stabilization contributes to $70 \%$ of KSI's catalytic power (Fig. S1B). ${ }^{1}$

In the following, we aim to provide a more complete and accurate description of electrostatic catalysis in KSI by including orientational effects. As shown in Fig. 1B, in addition to developing a bigger $\mathrm{C}=0$ dipole moment, $a b$ initio calculations on the isolated, full substrate in the gas phase suggest that the carbonyl bond of the substrate also undergoes a significant angle shift as the reaction proceeds (see SI Methods). Gas-phase optimized structures of the steroid ligand corresponding to the $\mathrm{GS}, \mathrm{TS}$, and dienolate intermediate suggest that a $\mathrm{C}=0$ dipole reorientation of up to $31^{\circ}$ could also be exploited by KSI to maximize the preferential electrostatic interaction with the TS (Fig. 1B). Because geometrical reorientation of the $\mathrm{C}=0$ dipole of the TS results mainly from the change in hybridization of the neighboring carbon (C4) from $\mathrm{sp}^{3}$ to $\mathrm{sp}^{2}$, we hypothesized that binding $5 \alpha$-dihydronandrolone (DHN) and $19 \mathrm{NT}$ to KSI could mimic the geometry of the enzyme:substrate complex in the GS and the TS/I states, respectively (Fig. 1A upper). We obtained crystal structures of the two inhibitors in complex with WT KSI to assess reorientation of the substrate's $\mathrm{C}=0$ dipole along the reaction coordinate. Furthermore, the electric fields experienced by the $\mathrm{C}=0$ of $19 \mathrm{NT}$ and DHN when bound to KSI were calculated with MD simulations from the crystallographic coordinates and estimated experimentally with VSE spectroscopy.
The difference in the electric fields experienced by the two ligands reflects the extent to which KSI's electric field is aligned with the TS's dipole.

The crystal structures of wild type KSI bound to DHN and bound to $19 \mathrm{NT}$ were obtained with a resolution of $1.7 \AA$. An overall RMS deviation of $0.181 \AA$ was observed when the two structures are superimposed, suggesting minimal change in the overall enzyme architecture within the error of the structure (Fig. 2A and statistics in Table S1). Focusing on the interactions between the ligands and two critical active site residues, Tyr ${ }^{16}$ and $\mathrm{Asp}^{103}$, the structures show that the positions of the active site residues are virtually unaltered between these two states, while the two ligands (most notably, the $\mathrm{C}=0$ bond) assume slightly different orientations relative to the protein. The carbonyl bond of DHN is shifted approximately $14^{\circ}$ "down" from that of $19 \mathrm{NT}$, smaller than the predicted difference of $31^{\circ}$ from the optimized gas phase geometries (Fig. 1B). Importantly, when the ligand geometries taken from the crystal structures are compared with the molecules' gas-phase optimal geometries, the carbonyl bond of both ligands are found to be distorted to more closely resemble the TS geometry (Fig. 2B and Fig. S2), explaining the smaller angle shift in KSI compared to in the gas phase.

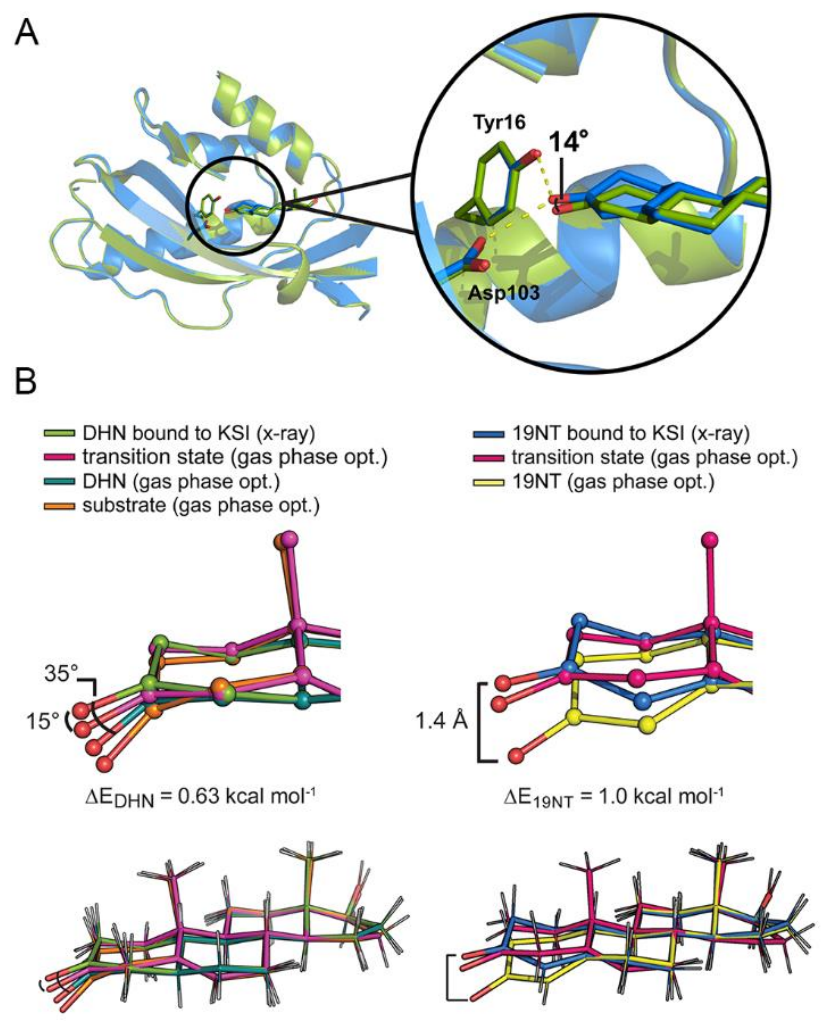

Figure 2. Crystal structures of wild type KSI bound with DHN (6UFS) and with 19NT (5KP4) show perturbed geometry of the ligands. (A) The structure of KSI bound to DHN (green, $1.7 \AA$ ) is globally aligned with that of KSI bound to 19NT (blue, $1.7 \AA$ ), giving an RMS deviation of $0.181 \AA$. Within this alignment frame, the angle shift between the carbonyl of DHN and the carbonyl of $19 \mathrm{NT}$ is about $14^{\circ}$, smaller than the predicted difference of $31^{\circ}$ from the optimized gas-phase geometries (Fig. 1B). (B) The $\mathrm{C}=0$ bond of DHN and 19NT gets distorted from their gas-phase optimal geometries to more closely resemble the TS structure when bound to KSI. A small energy penalty $(\Delta \mathrm{E})$ is calculated from the energy difference between the gas-phase 
optimal structure of the ligand and the gas-phase structure 'constrained' to the crystal coordinates (Fig. S4).

To ensure that the observed geometry distortion is not an artifact from refinement of the structure, we searched the Protein Data Bank for other crystal structures that contain DHN or highly similar molecules as a bound ligand and found two structures: $5 \alpha$-estran-3,7-dione (ESR) bound to KSI ${ }^{\mathrm{D} 40 \mathrm{~N}}$ from Comamonas testosteroni (an orthologue of the KSI we have studied herein, referred to as $t \mathrm{KSI}$, PDB 10HP) and dihydrotestosterone (DHT) bound to $17 \beta$-hydroxysteroid dehydrogenase type 1 (17 $\beta$-HSD1, PDB $3 \mathrm{KLM})$. We reasoned that if the perturbed geometry of the carbonyl groups in the KSI complexes is functionally relevant, it would be observed in the former structure but not the latter since $17 \beta$-HSD1 catalyzes a chemical conversion on the distal D-ring of the steroid, and has no activity for the carbonyl group on the A-ring. As shown in Fig. $\mathrm{S} 3 \mathrm{~A}$, the carbonyl group of ESR bound to $t \mathrm{KSI}$ is indeed distorted in a manner highly similar to that of DHN bound to KSI, while the geometry of $17 \beta$-HSD1 bound DHT resembles the gas-phase optimized geometry of DHN with mild structural alterations.

These observations are reminiscent of the 'Circe effect' proposed by Jencks, 12,13 who suggested that enzymes can destabilize a substrate's reactive region (in this case, the A-ring carbonyl bond) to facilitate its positioning in the enzyme's active site by utilizing favorable distal interactions (in this case, the multi-ring system) to offset the former's free energy cost.12 Using density functional theory (DFT) we estimated that the energy penalty for the bond distortion is close to $1.0 \mathrm{kcal} \mathrm{mol}^{-1}$ by comparing the energy of the gas-phase optimized geometry of the ligands (hereafter denoted as 'gas phase opt.') to the gasphase optimized energy of the ligands but in which the 4 dihedral angles of the A-ring are constrained to their values from the crystal structures ( $\Delta \mathrm{E}$ in Figure $2 \mathrm{~B})$. These distortion energies are very likely well below the energetic benefit that could be gained from forming an optimal $\mathrm{H}$-bond or electrostatic

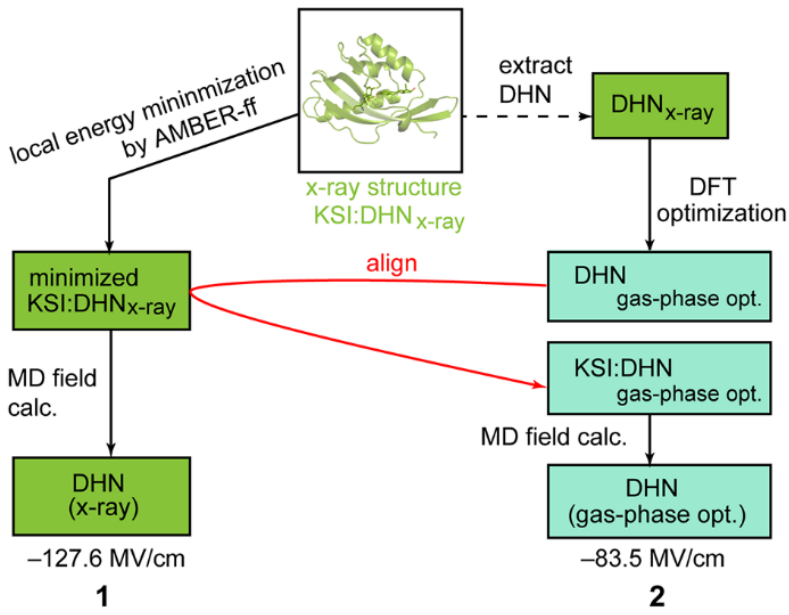

Figure 3. Modeling scheme of different KSI:DHN structures for MD calculations. Each pose is given a number whose corresponding simulation results are listed in Table 1 and Table S2. (See Fig. S4 for a more detailed scheme and numbers associated with other species).

interaction between the carbonyl and the oxyanion hole, as will be discussed in the following. Therefore, it is not surprising that KSI's large active site electric field partially aligns the carbonyl group of the substrate to maximize stabilization of the complex. The resulting TS-like geometry of the ligands thereby provides evidence that the electric field of KSI can orient the $\mathrm{C}=0$ to a TS-like geometry, and that this geometric preference is imposed upon a substrate-like ligand.

To test the hypothesis that the geometric perturbations experienced by steroids in KSI's active site are utilized for electrostatic catalysis, we carried out simulations using these crystal structures to estimate the electric field KSI projects on the $\mathrm{C}=\mathrm{O}$ bond dipole in each case and additionally calculated the alignment of the local electric field vector to the $\mathrm{C}=0$ bond vector with the equation:

$$
\text { \%aligned }=\vec{F}_{\text {enz }} \cdot \hat{u}_{\mathrm{co}} /\left|\vec{F}_{\text {enz }}\right|
$$

(details in SI Methods) where the numerator corresponds to the projection of the enzyme field onto the $\mathrm{C}=0$ bond unit vector, and the denominator is the magnitude of the enzyme field vector. ${ }^{14}$ The structures of KSI:19NT and KSI:DHN complexes were first energy minimized with the AMBER forcefield, and then a short simulation was run at low temperature on the energy-minimized complexes to derive the electric field magnitude at the carbonyl of $19 \mathrm{NT}$ or DHN and the field's projection along the $\mathrm{C}=0$ vector as previously described (denoted as ' $\mathrm{x}$ ray' in Table 1). ${ }^{14}$ These simulations were intentionally cold and short in order to associate an electric field with the crystallographic configuration, and are not intended to reflect a thermally-averaged value; 15 nevertheless, we ran simulations for ten 1 -fs steps to validate the structures were not unstable. ${ }^{15}$

Table 1 | Calculated active site electric fields and their geometry relative to the ligand's $\mathrm{C}=0$ bond vector ${ }^{a}$

\begin{tabular}{|c|c|c|c|c|c|}
\hline \multirow{2}{*}{$\begin{array}{c}\text { coordinates } \\
a\end{array}$} & \multicolumn{2}{|c|}{$\begin{array}{c}\text { DHN } \\
\text { (Substrate like) }\end{array}$} & \multicolumn{2}{|c|}{$\begin{array}{c}\text { 19NT } \\
\text { (TS like) }\end{array}$} & \multirow{2}{*}{$\begin{array}{c}\text { TS } \\
\text { gas } \\
\text { phase } \\
\text { opt. } \\
\text { (5) }\end{array}$} \\
\hline & $\begin{array}{l}\text { x-ray } \\
\text { (1) }\end{array}$ & $\begin{array}{l}\text { gas } \\
\text { phase } \\
\text { opt. } \\
(2)\end{array}$ & $\begin{array}{c}\text { x-ray } \\
(6)\end{array}$ & $\begin{array}{l}\text { gas } \\
\text { phase } \\
\text { opt. } \\
\text { (7) }\end{array}$ & \\
\hline $\begin{array}{c}\text { field } \\
\text { projection }^{b} \\
(\mathrm{MV} / \mathrm{cm})\end{array}$ & -127.6 & -83.5 & -141.7 & -76.1 & -144.6 \\
\hline \%aligned $c$ & $91 \%$ & $79 \%$ & $88 \%$ & $75 \%$ & $95 \%$ \\
\hline $\begin{array}{c}\Delta \text { stabiliza- }^{-} \\
\text {tion }^{d} \\
\left(\mathrm{kcal} \mathrm{mol}^{-1}\right)\end{array}$ & 6.6 & $\begin{array}{l}\text { refer- } \\
\text { ence }\end{array}$ & 10.7 & $\begin{array}{l}\text { refer- } \\
\text { ence }\end{array}$ & / \\
\hline $\begin{array}{c}\mathrm{C}=\mathrm{O} \ldots \mathrm{O}_{16} \\
(\AA)\end{array}$ & 2.67 & / & 2.57 & / & 2.70 \\
\hline $\begin{array}{c}\mathrm{C}=0 \ldots \mathrm{O}_{103} \\
(\AA))\end{array}$ & 2.73 & / & 2.65 & / & 2.48 \\
\hline
\end{tabular}

a See Table S2 and Fig. S4 for complete simulation scheme and results. The number of each entry corresponds to the respective species in Fig. 3 and Fig. S4

$b$ The electric field projected on the carbonyl is calculated by $\vec{F}_{e n z} \cdot \hat{u}_{C O}$

$c$ The \%aligned is calculated by Eq. 3

${ }^{d} \Delta$ stabilization (the stabilization energy gained from $\mathrm{C}=0$ distortion) is the difference of the field projection multiplied by the dipole of the carbonyl $\left(1 \mathrm{MV} \mathrm{cm}^{-1} \mathrm{D}^{-1} \simeq 0.048 \mathrm{kcal} \mathrm{mol}^{-1}\right)$

Next, we removed the DHN (or 19NT) coordinates from the xray structures, and replaced them with the DFT gas-phase optimized DHN (or 19NT) coordinates by alignment (Fig. 3 and 
also see Fig S4 for the full flowchart explaining these manipulations). These fictitious structures, denoted in Table 1 with the heading 'gas-phase opt.' coordinates, enable us to estimate how unfavorable the gas-phase optimal geometries are in the context of the electrostatic interactions created by KSI's active site.

As shown in Table 1, by dividing the projected field with the electric field magnitude (Eq. 3), we found that KSI's electric field is approximately $90 \%$ aligned with the carbonyl dipole of both DHN and 19NT. The \%aligned further increases to $95 \%$ when the modeled TS structure is docked into KSI by aligning it to 19NT (Fig. 3 and Fig. S4). Focusing on how the electric field is experienced by the carbonyl bond itself, the field projection on 19NT's carbonyl bond $(-141.7 \mathrm{MV} / \mathrm{cm})$ agrees well with that measured by VSE spectroscopy $(-141.3 \mathrm{MV} / \mathrm{cm}$, Table S4), though this agreement could be partially accidental since the calculations were not exhaustively sampled from a thermal distribution. ${ }^{1}$ The value is also close to the predicted field on the TS's C-O bond $(-144.6 \mathrm{MV} / \mathrm{cm})$, demonstrating $19 \mathrm{NT}$ to be a faithful probe for KSI's electrostatic environment in the TS because KSI perturbs 19NT's geometry to become very TS-like (Fig. 2B and Fig. S2). The field projection on DHN's carbonyl bond is $14 \mathrm{MV} / \mathrm{cm}$ smaller than that of $19 \mathrm{NT}(-127.6 \mathrm{MV} / \mathrm{cm})$, which can be explained in part because the $0-0$ distances between the carbonyl of DHN and the hydroxyl group of Tyr16 and Asp103 are both slightly longer $(\sim 0.1 \AA)$ than their counterparts in the $19 \mathrm{NT}$ complex. ${ }^{16}$

As seen in Table 1, the electric fields calculated based on the ligands' actual geometries when bound to KSI are considerably larger than those based on the gas-phase optimized geometries (also see Table S2 and discussion S1). These calculations though based on fictitious structures - suggest a significant energetic benefit associated with bringing the $\mathrm{C}=0$ dipole deep in the active site and aligned with KSI's electric field. We estimate these structural rearrangements strengthen the enzyme's electrostatic interaction with DHN by $\sim 6 \mathrm{kcal} \mathrm{mol}^{-1}$ and with $19 \mathrm{NT}$ by $\sim 11 \mathrm{kcal} \mathrm{mol}^{-1}$ - more than enough to compensate for the small energetic cost of $\sim 1 \mathrm{kcal} \mathrm{mol}^{-1}$ from local ligand bond distortion. These results imply that despite what may superficially appear to be a distortion from inspection of the structure, KSI's binding mode of its substrate in fact stabilizes the $\mathrm{C}=0$ dipole in both the GS and TS structures, in contradistinction to the Circe effect (Fig. S5).

The calculations above using crystallographic structures point to an electrostatic environment in KSI's active site that is pre-organized towards the TS geometry. Upon binding to KSI, the substrate-like ligand is driven by a large, oriented electric field to assume a TS-like geometry at the reactive site, so that minimal dipole reorientation needs to occur during the reactive event.

Using the projected fields on the carbonyl of $19 \mathrm{NT}$ and DHN (Table 1), we can estimate the partial contribution of the orientational effect to the total electrostatic stabilization of KSI, described at the outset. The total stabilization estimated using Eq. 1 is:

$$
\begin{gathered}
\Delta \Delta G^{\ddagger}=-\left(\left(\vec{F}_{\mathrm{enz}, \mathrm{TS}} \cdot \vec{\mu}_{\mathrm{TS}}\right)-\left(\vec{F}_{\mathrm{enz}, \mathrm{R}} \cdot \vec{\mu}_{\mathrm{R}}\right)\right) \\
=-((142 \mathrm{MV} / \mathrm{cm} \times 4.1 \mathrm{D})-(128 \mathrm{MV} / \mathrm{cm} \times 3.4 \mathrm{D})) \\
=-7.1 \mathrm{kcal} / \mathrm{mol}
\end{gathered}
$$

noting that $1 \mathrm{MV} \mathrm{cm}^{-1} \mathrm{D}^{-1}$ is approximately $0.048 \mathrm{kcal} \mathrm{mol}^{-1}$. In contrast, the stabilization energy if only the scaling effect was considered estimated using Eq. 2 is:

$$
\Delta \Delta G^{\ddagger}=-\left|\vec{F}_{\mathrm{enz}}\right|\left(\left|\vec{\mu}_{\mathrm{TS}}\right|-\left|\vec{\mu}_{\mathrm{R}}\right|\right)
$$

$$
\begin{gathered}
=-142 \mathrm{MV} / \mathrm{cm} \times(4.1-3.4) \mathrm{D} \\
=-4.8 \mathrm{kcal} / \mathrm{mol}
\end{gathered}
$$

The angular preference of the active site electric field favoring the TS geometry therefore imparts an additional stabilization energy of $2.3 \mathrm{kcal} / \mathrm{mol}$, corresponding to $30 \%$ of the total electrostatic rate acceleration. Based on this simple analysis, KSI is able to selectively stabilize the TS significantly more than the GS by exploiting a fairly small shift in $\mathrm{C}=0$ orientation. This notion of 'geometric discrimination' has been previously discussed $^{20}$, though is treated here quantitatively within an electrostatic framework. In this context, we might refer to the active site electric field as 'pre-organized' because: (i) it optimally stabilizes the TS geometry; (ii) it does not change to accommodate the GS geometry (as a bulk solvent would), but rather forces the GS to assume a more 'TS-like' geometry. Note that, the catalytic effect ascribed to electrostatics here is quite similar to the value we estimated previously $\left(7.3 \pm 0.4 \mathrm{kcal} \mathrm{mol}^{-1}\right)^{6}$, though importantly, here we have obtained the result without any extrapolations (also see discussion S2). ${ }^{17}$

The preorganization of the electric field in enzymes highlights its fundamental difference from the solvent reaction field in aqueous solutions, which is instead optimized for the GS charge distribution and has to reorient to accommodate the TS as the reaction proceeds, imposing a reorganization energy. ${ }^{4}$ The energetic cost of solvent reorganization limits the catalytic capacity of water despite the significant magnitude of its electric field (Fig. S5).4,18

We sought to further validate this notion experimentally by measuring the electric field projection on the carbonyl of DHN via VSE spectroscopy, under which conditions the enzyme-ligand interaction is fully equilibrated. In order to obtain reliable IR spectra of the carbonyl group in the midst of the strong background from the protein amide band I, it is essential to obtain isotope-edited difference spectra, as was done for 19NT previously. ${ }^{1}$ While it is straightforward to prepare the ${ }^{18} 0$-substituted version of DHN, rapid back-exchange with bulk water, even when the sample was prepared in $\mathrm{D}_{2}{ }^{18} \mathrm{O}$ (and the protein uniformly ${ }^{13} \mathrm{C}$ labeled to shift the amide I band), made it difficult to obtain definitive data (in contrast, back-exchange for $19 \mathrm{NT}$ is very slow). The results of our attempts to perform this measurement are presented in discussion S3 and Fig. S6.

In summary, this work clarifies a number of important aspects about electrostatic catalysis. Perturbed substrate geometry has long been considered a key factor in promoting catalysis, ${ }^{19-21}$ however the belief that such distortions implied a ground-state destabilization contribution could not be easily reconciled with computation and theory. ${ }^{22}$ Here we show that the catalytic effects associated with altered substrate geometry are best interpreted within an electrostatic framework, where they serve to prime a substrate for movement to a transition state, and which can nevertheless be stabilizing in the ground state thanks to large active site electric fields. In KSI, this orientational effect provides a significant catalytic contribution, which is impressive in that the geometric changes during this reaction are actually quite small. The benefits of a preorganized electrostatic environment in enzyme catalysis would be expected to be even more profound in cases where a substrate's geometrical reorientation along the reaction coordinate are more dramatic, as is the case in many other enzymatic transformations.

\section{ASSOCIATED CONTENT}

\section{Supporting Information}


Experimental procedures, crystallographic data, complete information on the computational modeling scheme and results, raw IR spectra of DHN, and further evaluation on the energy profile of the isomerization reaction in KSI and in solution are included in the supporting information. This material is available free of charge via the internet at http://pubs.acs.org.

\section{AUTHOR INFORMATION}

\section{Corresponding Author}

*Correspondence should be addressed to: sboxer@stanford.edu

\section{Present Addresses}

† Current Address: Department of Biological Chemistry and Molecular Pharmacology, Harvard Medical School, Boston, Massachusetts 02115, United States

‡ Current Address: Department of Chemistry, Johns Hopkins University, Baltimore, Maryland 21218, United States

\section{Notes}

The authors declare no competing financial interests.

\section{ACKNOWLEDGMENT}

This work was supported, in part, by NIH Grant GM118044 (to S.G.B.). Use of the Stanford Synchrotron Radiation Lightsource (SSRL), SLAC National Accelerator Laboratory, is supported by the U.S. Department of Energy, Office of Science, Office of Basic Energy Sciences under Contract No. DE-AC02-76SF00515. The SSRL Structural Molecular Biology Program is supported by the DOE Office of Biological and Environmental Research, and by the National Institutes of Health, National Institute of General Medical Sciences (including P41GM103393). S.D.F. was supported by a Junior Research Fellowship from King's College, Cambridge (UK) at the time of this work. The contents of this publication are solely the responsibility of the authors and do not necessarily represent the official views of NIGMS or NIH.

\section{REFERENCES}

(1) Fried, S. D.; Bagchi, S.; Boxer, S. G. Science 2014, 346, 15101513.

(2) Wu, Y.; Boxer, S. G. J. Am. Chem. Soc. 2016, 138, 11890-11895.

(3) Radzicka, A.; Wolfenden, R. Science 1995, 267, 90-93.

(4) Warshel, A.; Sharma, P. K.; Kato, M.; Xiang, Y.; Liu, H.; Olsson, M. H. M. Chem. Rev. 2006, 106, 3210-3235.

(5) Hanoian, P.; Liu, C. T.; Hammes-Schiffer, S.; Benkovic, S. Acc. Chem. Res. 2015, 48, 482-489.

(6) Fried, S. D.; Boxer, S. G. Annu. Rev. Biochem. 2017, 86, 387-415.

(7) Zoi, I.; Antoniou, D.; Schwartz, S. D. J. Phys. Chem. Lett. 2017, 8, 6165-6170.

(8) Herschlag, D.; Natarajan, A. Biochemistry 2013, 52, 20502067.

(9) Menger, F. M.; Nome, F. ACS Chem. Biol. 2019, 14, 1386-1392.

(10) Pollack, R. M. Bioorg. Chem. 2004, 32, 341-353.

(11) Fafarman, A. T.; Sigala, P. A.; Schwans, J. P.; Fenn, T. D.; Herschlag, D.; Boxer, S. G. Proc. Natl. Acad. Sci. U. S. A. 2012, 109, E299-308.

(12) Jencks, W. P. Anvances Enzymol. Relat. Subj. 1975, 43, 219410.

(13) Page, M. I.; Jencks, W. P. Proc. Natl. Acad. Sci. USA 1971, 68, 1678-1683.

(14) Fried, S. D.; Bagchi, S.; Boxer, S. G. J. Am. Chem. Soc. 2013, 135, 11181-11192.

(15) Welborn, V. V.; Head-gordon, T. J. Am. Chem. Soc. 2019, 141, 12487-12492.

(16) Wang, L.; Fried, S. D.; Markland, T. E. J. Phys. Chem. B 2017, 121, 9807-9815

(17) Natarajan, A.; Yabukarski, F.; Lamba, V.; Schwans, J. P.; Sunden, F.; Herschlag, D. Science 2015, 349, 936

(18) Fried, S. D.; Boxer, S. G. Science 2015, 349, 936.

(19) Blake, C. C.; Johnson, L. N.; Mair, G. A.; North, A. C.; Phillips, D. C.; Sarma, V. R. Proc. R. Soc. London B Biol. Sci 1967, 167, 378388.

(20) Robertus, J. D.; Kraut, J.; Alden, R. A.; Birktoft, J. J. Biochemistry 1972, 11, 4293-4303.

(21) Fastrez, J.; Fersht, A. R. Biochemistry 1973, 12, 2025-2034.

(22) Warshel, A. J. Biol. Chem. 1998, 273, 27035-27038. 


\section{TOC Graphic}

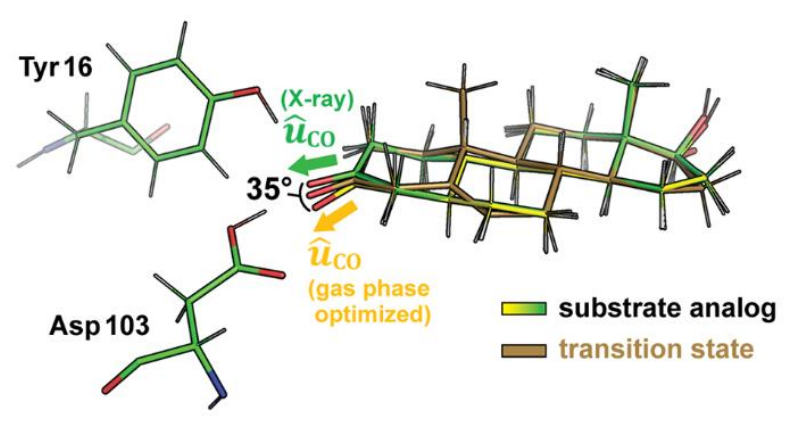




\title{
Supporting information for:
}

\section{"A Preorganized Electric field leads to Minimal Geometrical Reorientation in the Catalytic Reaction of Ketosteroid Isomerase"}

\author{
Yufan Wu, Stephen D. Fried and Steven G. Boxer \\ Department of Biological Chemistry and Molecular Pharmacology, Harvard Medical School, \\ Boston, Massachusetts 02115, United States \\ Department of Chemistry, John Hopkins University, Baltimore, Maryland 21218, United States \\ Department of Chemistry, Stanford University, Stanford, California 94305-5012, United States
}

\section{$\underline{\text { Contents }}$}

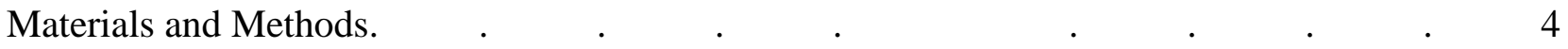

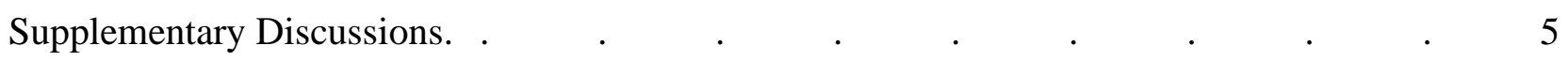

1. Further evaluation of the modelling scheme of KSI complexes in MD simulations. 5

2. Further discussions on the estimation of orientational effect. $\quad$. $\quad$. $\quad$. 7

3. Vibrational Stark Effect Spectroscopy studies on DHN and KSI:DHN. . . . 7

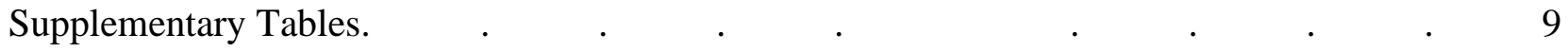

1. X-ray diffraction data collection and refinement statistics. . $\quad$. $\quad$. $\quad$. $\quad$. $\quad 9 \quad 9$

2. KSI active site electric fields from structural analysis and MD calculations. $\quad 10$

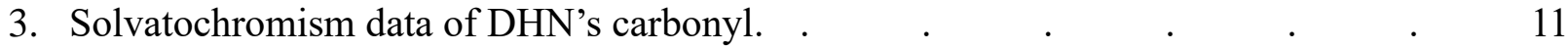

4. Electric fields of ligands bound to KSI as determined by Vibrational Stark Effects . 11

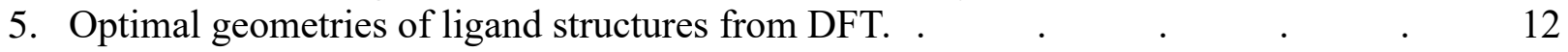

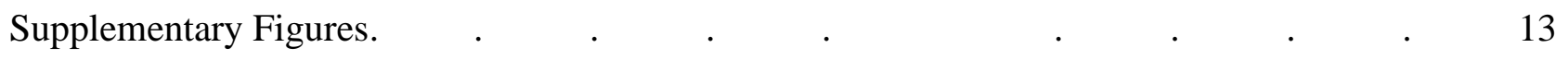

1. Linear correlation between KSI's electric field and rate acceleration _ . . $\quad 13$

2. Electron density maps of the oxyanion hole residues and the bound ligand showing their relative positioning.

3. Geometrical change between steroids bound to enzymes and in the gas phase. $\quad$. $\quad 15$

4. Modelling scheme of different KSI:ligand complexes for MD calculations. . 16

5. Stabilization energy in different environments and the free energy profiles of the isomerization reaction in KSI and in aqueous solution. $\quad . \quad$. 17

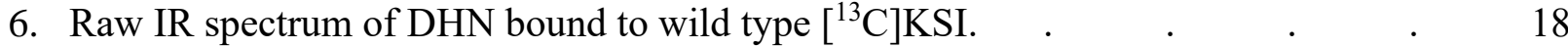

7. ${ }^{1}$ H-NMR spectrum of purified dihydronandrolone sulfate. . . . . . . . . 19 
Materials and Methods.

Materials. 5 $\alpha$-Dihydronandrolone (DHN) was purchased from Steraloids (Newport, RI). Anhydrous solvents and deuterium oxide $(99.5 \%$ D) were purchased from Acros Organics and Cambridge Isotopes. Sulfur trioxide-pyridine complex was purchased from Sigma-Aldrich. ${ }^{13} \mathrm{C}_{6}$ D-glucose (99\%), $\mathrm{H}_{2}{ }^{18} \mathrm{O}(97 \%)$ and $\mathrm{D}_{2}{ }^{18} \mathrm{O}(98 \% \mathrm{D}, 97 \% \mathrm{O})$ were purchased from Cambridge Isotopes.

Preparation of uniformly ${ }^{\mathbf{1 3}} \mathbf{C}$ labeled KSI. Preparation of wild type KSI was carried out using BL21(AI) cells (Invitrogen) in M9 minimal medium, augmented with $1 \mathrm{mM} \mathrm{MgSO}_{4}, 0.1 \mathrm{mM}$ $\mathrm{CaCl}_{2}, 0.2 \%{ }^{13} \mathrm{C}_{6}$-D-glucose and MEM Vitamin Solution. Protein expression was induced at OD 0.8 with $1 \mathrm{mM}$ IPTG and the cells were grown for another 7-9 hours at $37^{\circ} \mathrm{C}$ before harvest. The protein was purified with Ni-NTA affinity chromatography and anion exchange chromatography (GE Healthcare) as described previously. ${ }^{1}$

Synthesis and purification of 5 $\alpha$-Dihydronandrolone sulfate. $10 \mathrm{mg}$ of $5 \alpha$-Dihydronandrolone (DHN) was dissolved in $160 \mu \mathrm{L}$ of dimethylformamide and reacted with $30 \mathrm{mg}$ of sulfur trioxidepyridine complex. The reaction vial was flushed with nitrogen gas for 3-5 minutes to eliminate unwanted moisture and sealed. The reaction was allowed to run overnight under constant stirring. The progress of the reaction was monitored with thin-layer chromatography (EtOAc : $\mathrm{MeOH}$ : $\left.\mathrm{H}_{2} \mathrm{O}, 7: 2: 1, \mathrm{v} / \mathrm{v}\right)$. Due to the small scale of the reaction, the product DHN sulfate was isolated using reverse phase high performance liquid chromatography (RP-HPLC) on a C18 column with water : acetonitrile (15:85, buffered with $5 \mathrm{mM}$ ammonium acetate at $\mathrm{pH}=6.5)$ as the mobile phase. The retention time of the DHN sulfate was 28 minutes (at $45 \% \mathrm{~B}$ ). A small peak corresponding to the unreacted $\mathrm{DHN}$ was observed at 38 minutes $(75 \% \mathrm{~B})$. The purity of the isolated DHN sulfate was assessed via ${ }^{1} \mathrm{H}-\mathrm{NMR}$ (Fig. S7).

Isotopic labeling of $5 \boldsymbol{\alpha}$-dihydronandrolone sulfate. To make $\left[{ }^{18} \mathrm{O}\right] \mathrm{DHN}$ sulfate, about $1.0 \mathrm{mg}$ of DHN sulfate was dissolved in $50 \mu \mathrm{L}$ of $\mathrm{D}_{2}{ }^{18} \mathrm{O}$. The reaction vial (Eppendorf microcentrifuge tube) was sealed and the reaction was allowed to proceed at room temperature overnight. The labeling reaction of the carbonyl was found to proceed readily without the need of acetic acid as a catalyst. However, the reverse reaction was also found to gradually occur during the course of IR sample preparation and measurements when trace amount of $\mathrm{H}_{2}{ }^{16} \mathrm{O}$ were present, preventing unambiguous detection and assignment of the DHN carbonyl peak signals (see text), in contrast to earlier work with $\left[{ }^{18} \mathrm{O}\right] 19 \mathrm{NT} .{ }^{2}$ 
X-ray crystallography. Crystals of wild type KSI bound with $5 \alpha$-dihydronandrolone and with 19nortestosterone were obtained using hanging drop vapor diffusion. $1 \mu \mathrm{L}$ of $1 \mathrm{mM} \mathrm{KSI}$ (in $40 \mathrm{mM}$ $\mathrm{KPi}, \mathrm{pH}$ 7.2), preincubated with $2 \mathrm{mM}$ ligand, was mixed with $1 \mu \mathrm{L}$ of reservoir solution (1.0-1.2 $\mathrm{M}$ ammonium sulfate, $40 \mathrm{mM} \mathrm{KPi}(\mathrm{pH} \mathrm{7.2),} \mathrm{1-3 \%} \mathrm{isopropanol).} \mathrm{The} \mathrm{hanging} \mathrm{drops} \mathrm{were}$ equilibrated for 1-2 days before microseeding to initiate the crystallization. Microseeding was performed by crushing low quality crystals of apo $\mathrm{KSI}-\mathrm{ClY}^{16}$ variant $^{1}$ by vortexing with a glass bead, serially diluting the resulting nanocrystals (5000-10000 times), and using a cat whisker to transfer the microseeds to the equilibrated drops. ${ }^{3,4}$ Cryoprotection was achieved by directly soaking crystals in $2.0 \mathrm{M}$ sucrose prior to flash freezing in liquid nitrogen. Single crystal diffraction data were collected at the Stanford Synchrotron Radiation Laboratory BL12-2. Data were integrated and scaled using HKL2000 for the DHN structure and XDS for the 19NT structure, respectively. ${ }^{5-7}$ Data collection and refinement statistics are summarized in Table S1. An initial protein model for each structure was obtained by molecular replacement with Phaser $^{8}$ using the coordinates from the previously published structure of apo KSI-D40N-ClY ${ }^{57}$ (PDB 5D81). Apo KSI was chosen as the initial model to eliminate potential model bias in the ligand binding geometry. Simulated annealing refinement was carried out using a maximum-likelihood amplitude-based target function as implemented in Phenix ${ }^{9}$. Further refinement was carried out with Phenix, interspersed with manual model building in $\operatorname{Coot}^{10}$. All structural figures were prepared using PyMOL ${ }^{11}$.

FTIR spectroscopy. FTIR spectra were obtained on a Bruker Vertex 70 spectrometer with a liquid nitrogen-cooled mercury cadmium telluride (MCT) detector using methods very similar to those described previously. ${ }^{12}$ The concentration of the DHN sulfate stock was calibrated against known concentrations of trimethylsilylpropanoic acid (TSP-d 4$)$ standard and $19 \mathrm{NT}$ sulfate solution $\left(\varepsilon_{248}\right.$ $=14.6 \mathrm{mM}^{-1} \mathrm{~cm}^{-1}$ ) with ${ }^{1} \mathrm{H}-\mathrm{NMR}$. An appropriate amount of each ligand stock was added to the protein aliquot in the same solvent. The final liquid solution contains around $4 \mathrm{mM}$ protein and 3 $\mathrm{mM}$ ligand. Spectra of the sample and the reference were acquired following a 10 minutes nitrogen purge and averaging over 512 scans. In our attempt to measure the IR signals using [ $\left.{ }^{18} \mathrm{O}\right] \mathrm{DHN}$ sulfate bound to KSI as the spectral reference, two equal amount of KSI samples were prepared first in $10 \mathrm{mM} \mathrm{KPi}$ buffer (in $\mathrm{H}_{2}{ }^{16} \mathrm{O}$ ) and lyophilized. One sample was then dissolved in $\mathrm{D}_{2} \mathrm{O}$ while the other in $\mathrm{D}_{2}{ }^{18} \mathrm{O}$. Ligand stocks of $\left[{ }^{16} \mathrm{O}\right] \mathrm{DHN}$ sulfate or $\left[{ }^{18} \mathrm{O}\right] \mathrm{DHN}$ sulfate were directly prepared at around $50 \mathrm{mM}$ in $\mathrm{D}_{2} \mathrm{O}$ or $\mathrm{D}_{2}{ }^{18} \mathrm{O}$.

Solvatochromism of DHN and DHN sulfate. The solvatochromism model for DHN was constructed using a previous method. ${ }^{2,13}$ In brief, the vibrational frequencies of the carbonyl bond of DHN dissolved in various organic solvents and of DHN sulfate in water were measured. Next, the electric field exerted onto the carbonyl bond by each solvation environment was calculated 
from MD simulations in GROMACS with the generalized AMBER force field (GAFF). ${ }^{13}$ By plotting the calculated electric field in the simple solvents (solvent field) against the IR peak positions of DHN, a linear correlation between the absolute electric field and the carbonyl's vibrational frequency was established to be $\bar{v}_{\mathrm{C}=\mathrm{O}}=0.484\left|\vec{F}_{\text {solv }}\right|+1726$. Both DHN and DHN sulfate were dissolved in DMSO and their carbonyl vibrational frequencies were found to be identical showing that remote sulfylation of the ligand did not affect the $\mathrm{C}=\mathrm{O}$ 's vibrational frequency. The correspondence from the solvatochromism data was then used to map the measured IR peak(s) of the $\mathrm{C}=\mathrm{O}$ bond of DHN sulfate in wild type KSI to the electric field the enzyme projects on the $\mathrm{C}=\mathrm{O}$ bond axis.

\section{Ligand geometry optimization with DFT and electric field modeling with AMBER force}

field. For each ligand, two or three different geometries are modeled. The optimal geometries were obtained from gas-phase DFT calculations at the B3LYP/6-311++G(d,p) level (Table S5) of the isolated steroid molecules (substrate (5AND), DHN (substrate mimic), intermediate (I), 19NT (I/TS mimic)). All DFT calculations were carried out in Gaussian 03. The geometry of the TS was determined by Berny optimization starting from 5AND as described previously, ${ }^{2}$ and additionally included an acetate molecule to serve as the proton acceptor of 5AND's $4 \beta$ proton. The constrained geometries of DHN and 19NT were obtained in the same way except the 4 dihedral angles of the A-ring of the ligand were fixed to those from the crystal structure. To constrain the dihedral angles of substrate (5AND), the dihedral angles from the KSI:DHN structure were used; to constrain the dihedral angles of the TS and intermediate (I), the dihedral angles from the KSI:19NT structure were used. The energy costs for the carbonyl angle shift observed in the Xray structures of $19 \mathrm{NT}$ and DHN (Fig. 2) were estimated as the energy difference between the optimal geometry and the constrained geometry (bond dipole moments were calculated as described previously ${ }^{2}$ ).

Electric field calculations were carried out using the AMBER99SB-ILDN force field with the GROMACS package. As shown in Fig. S4, we started with the X-ray coordinates of the KSI:19NT and KSI:DHN complexes, immersed them in a $10 \mathrm{~nm}^{3}$ cubic box of TIP3P water using the genbox utility, and energy minimized by steepest descent. The structural model of KSI bound with the TS, I, or substrate was constructed by first aligning the TS and I to 19NT or substrate to DHN in the corresponding KSI:19NT and KSI:DHN energy-minimized structure and then exchanging the aligned coordinates of the molecule back into the same crystal structure. Short simulations at a temperature of 1 Kelvin were then ran for 10 steps on the structural model. Lastly, the total electric field magnitude at the $\mathrm{C}=\mathrm{O}$ of the ligand and the field projection along the $\mathrm{C}=\mathrm{O}$ vector were derived as previously described, ${ }^{13}$ and the \%aligned was estimated by dividing the latter with the former. The various ligands were parametrized using the same strategy as in the solvatochromism 
study. ${ }^{13}$ We note that the final answer obtained for the electric field from the environment is mostly invariant to the details of the ligand parameters since we subtract away the self-field.

\section{Supplementary discussions}

\section{Further evaluation of the modelling scheme of KSI complexes in MD simulations}

Apart from DHN, 19NT and the TS, we also performed MD simulations on the KSI:substrate complex and the KSI:intermediate complex. As we cannot obtain direct structural data of KSI:substrate and KSI:intermediate (they are turned over on the sub-ms timescale), we have modeled the structure of KSI:substrate by optimizing the substrate structure with a gas-phase DFT calculation, but subject to the constraint that the A ring adopts the perturbed geometry observed in the KSI:DHN x-ray structure, and then replacing the DHN coordinates with those of the substrate by aligning them to DHN coordinates (Fig. S4). This is referred to as the 'constrained' geometry in Table S2, and corresponds to our best estimate of what the substrate geometry looks like when bound to KSI. Likewise, for the intermediate, we carried out gas-phase DFT calculations subject to the constraint that the intermediate adopts the perturbed geometry observed in the KSI:19NT x-ray structure, and then replaced the 19NT coordinates with those of the intermediate by aligning them to 19 NT coordinates (Fig. S4). As shown in Table S2, when the substrate adopts the same perturbed geometry as in DHN ('constrained'), the carbonyl bond is better aligned with the enzyme's electric field vector, resulting in a much larger projection. This result is very similar to that of DHN itself, for which actual x-ray coordinates (rather than coordinates from a constrained optimization) were used. In contrast, when the intermediate is constrained to adopt the same perturbed geometry as in 19NT, the electric field it experiences is virtually the same as if its gas-phase optimized structure were used. These results further point to the notion that the electric field in KSI is pre-oriented towards the TS/intermediate geometry. Upon substrate binding, this large pre-oriented electric field distorts the carbonyl bond of the substrate to position it towards a TS-like geometry. However, the resulting rearrangement is expected to still result in overall stabilization because of the improved alignment with the electric field.

To further validate our modeling scheme, we also compared the MD calculated stabilization energy of binding the ligand to KSI from solution with that derived from the binding/rate constants (Fig. S5). Empirically, based on the dissociation constant of the substrate $\left(K_{\mathrm{D}}{ }^{\mathrm{S}}\right)^{14}$ and the microscopic rate constant of the reaction in enzyme ${ }^{15}$ and in solution, ${ }^{16}$ the substrate and the TS are stabilized by $6.6 \mathrm{kcal} \mathrm{mol}^{-1}$ and $18.2 \mathrm{kcal} \mathrm{mol}^{-1}$ respectively upon binding to KSI. Invoking Marcus theory, we estimate the stabilization energy of the TS in water under the assumption that the solvent reaction field is equilibrated with the reactant's dipole orientation (which is $31^{\circ}$ out of alignment with the TS, Fig. 1B). The stabilization energy in KSI is estimated from the MD calculated electric field projections based on the modeled structures of the KSI:substrate and 
KSI:TS complexes (Table S2 in bold). As shown in Figure S5, KSI's electrostatic environment is predicted to stabilize the substrate by $6.1 \mathrm{kcal} \mathrm{mol}^{-1}$ and the TS by $15.9 \mathrm{kcal} \mathrm{mol}^{-1}$ (dotted red lines) relative to water, in qualitative agreement with the experimental estimations. This provides further support for the semi-quantitative analysis presented in the main text.

\section{Further discussions on the estimation of orientational effect}

In order to quantify the total preferential stabilization of the TS over the GS from KSI's electric field, we employed equation (1):

$$
\Delta \Delta G^{\ddagger}=-\left(\left(\vec{F}_{\mathrm{enz}, \mathrm{TS}} \cdot \vec{\mu}_{\mathrm{TS}}\right)-\left(\vec{F}_{\mathrm{enz}, \mathrm{R}} \cdot \vec{\mu}_{\mathrm{R}}\right)\right)
$$

We reason that the binding modes of 19NT and DHN to KSI mimic that of the substrate and the TS based on their $\mathrm{C}_{4}$ hybridizations, therefore, equation (1) is converted to:

$$
\begin{aligned}
& =-\left(\left(\left(\vec{F}_{\mathrm{enz}}^{19 \mathrm{NT}} \cdot \hat{\mu}_{\mathrm{C}=0}^{19 \mathrm{NT}}\right)\left|\vec{\mu}_{\mathrm{C}=0}^{\mathrm{TS}}\right|\right)-\left(\left(\vec{F}_{\mathrm{enz}}^{\mathrm{DHN}} \cdot \hat{\mu}_{\mathrm{C}=0}^{\mathrm{DHN}}\right)\left|\vec{\mu}_{\mathrm{C}=0}^{5 \mathrm{AND}}\right|\right)\right) \\
& =-(((142 \mathrm{MV} / \mathrm{cm}) \times 4.1 \mathrm{D})-((128 \mathrm{MV} / \mathrm{cm}) \times 3.4 \mathrm{D})) \\
& =-7.1 \mathrm{kcal} / \mathrm{mol}^{1}
\end{aligned}
$$

where $\left(\vec{F}_{\text {enz }}^{19 N T} \cdot \hat{\mu}_{C=0}^{19 N T}\right)$ is the projection of KSI's electric field along the direction of 19NT's carbonyl bond and is determined by calculating the electric field of the enzyme from MD simulations that started with the KSI:19NT crystal structure coordinates. Likewise, $\left(\vec{F}_{\mathrm{enz}}^{\mathrm{DHN}} \cdot \hat{\mu}_{\mathrm{C}=0}^{\mathrm{DHN}}\right)$ is the projection of KSI's electric field along the direction of DHN's carbonyl bond and is determined by calculating the electric field of the enzyme from MD simulations that started with the KSI:DHN crystal structure coordinates (Table S2). Alternatively, KSI's projected field could also be derived from the vibrational frequency of the carbonyl bond via VSE spectroscopy, where the field projection on the carbonyl vector is intrinsic in the experimental measurement (Table S4, Fig. S5). $\left|\vec{\mu}_{C=0}^{\mathrm{TS}}\right|$ and $\left|\vec{\mu}_{\mathrm{C}=0}^{5 \mathrm{AND}}\right|$ are the magnitudes of the $\mathrm{C}=\mathrm{O}$ dipole of the two ligands and are calculated from the gas-phase optimized structures of the TS and the substrate from ab initio calculations (Fig. 1B).

To quantify the preferential stabilization of the TS over the GS from a pure scaling effect using our previous model ${ }^{2,12}$, the projection of KSI's electric field on the reactive carbonyl bond of the ligand is held constant while the magnitude of the bond dipole changes:

$$
\begin{aligned}
\Delta \Delta G^{\ddagger} & =-\left(\left(\vec{F}_{\mathrm{enz}, \mathrm{TS}} \cdot \vec{\mu}_{\mathrm{TS}}\right)-\left(\vec{F}_{\mathrm{enz}, \mathrm{R}} \cdot \vec{\mu}_{\mathrm{R}}\right)\right) \\
& =-\left(\vec{F}_{\mathrm{enz}} \cdot \hat{\mu}_{\mathrm{C}=0}\right)\left(\left|\vec{\mu}_{\mathrm{TS}}\right|-\left|\vec{\mu}_{\mathrm{R}}\right|\right) \\
& =-\left|F_{\mathrm{enz}}\right|\left(\left|\vec{\mu}_{\mathrm{TS}}\right|-\left|\vec{\mu}_{\mathrm{R}}\right|\right)
\end{aligned}
$$

${ }^{1} 1 \mathrm{MV} / \mathrm{cm}=0.048 \mathrm{kcal} \mathrm{mol}^{-1} \mathrm{D}^{-1}$ 


$$
\begin{aligned}
& =-142 \mathrm{MV} / \mathrm{cm} \times(4.1-3.4) \mathrm{D} \\
& =-4.8 \mathrm{kcal} / \mathrm{mol}
\end{aligned}
$$

Therefore, the inclusion of the orientational effect in our description of electrostatic catalysis in KSI reveals an additional $30 \%$ preferential stabilization energy in the total rate acceleration.

We note that in our previous model, we derived a value of $\left(\left|\vec{\mu}_{\mathrm{TS}}\right|-\left|\vec{\mu}_{\mathrm{R}}\right|\right)$ for the $\mathrm{C}=\mathrm{O}$ dipole

of $1.1 \mathrm{D}$ by inverting equation (2) using experimental values for $\Delta \mathrm{G}^{\ddagger}$ and $\left|F_{\text {enz }}\right|$ (Fig. S1B). ${ }^{2,12}$ This value is $0.4 \mathrm{D}$ larger than the predicted value from $a b$ initio calculations. The discrepancy can be explained in that by fitting rate data to equation (2), when equation (1) is the more correct model, the orientational effect became packaged into $\left(\left|\vec{\mu}_{\mathrm{TS}}\right|-\left|\vec{\mu}_{\mathrm{R}}\right|\right)$, resulting in a larger value. On the other hand, the ab initio values for $\left|\vec{\mu}_{\mathrm{TS}}\right|$ and $\left|\vec{\mu}_{\mathrm{R}}\right|$ used in this study can be seen as lower limits, as these dipoles would be expected to be larger in the enzyme active site due to polarization.

\section{Vibrational Stark Effect Spectroscopy studies on DHN and KSI:DHN}

We first calibrated the sensitivity of DHN's $\mathrm{C}=\mathrm{O}$ vibrational frequency to electric field. Analogous to previous studies on 19NT, the Stark tuning rate of the carbonyl group of DHN is calibrated using solvatochromism. ${ }^{2,12}$ Due to the limited solubility of DHN in water, its sulfate version (DHN sulfate) was synthesized and used to determine the carbonyl's vibrational frequency in water. As shown in Fig. S6A, a systematic shift to the red is observed for DHN's carbonyl frequency in solvents with increasing polarity. The average value of the solvent field experienced by the carbonyl is calculated from MD simulations following a previous protocol (Table S3). ${ }^{13}$ When the average value of the solvent field is plotted against DHN's carbonyl frequency, a linear correlation is observed giving a calibration curve of $\bar{v}_{\mathrm{C}=\mathrm{O}}\left(\mathrm{cm}^{-1}\right)=1726.5+0.484 \times \vec{F}_{\text {solv }}$ $(\mathrm{MV} / \mathrm{cm})$, where $0.484 \mathrm{~cm}^{-1} /(\mathrm{MV} / \mathrm{cm})$ is the Stark tuning rate $\left(\left|\Delta \vec{\mu}_{D H N}^{C=O}\right|\right)$. The sensitivity of DHN's carbonyl frequency to the electric field is smaller than that of $19 \mathrm{NT}$, agreeing with previous findings that conjugated carbonyls tend to exhibit larger Stark tuning rates. ${ }^{17}$

To determine the electric field experienced by the carbonyl of DHN when bound to KSI, we prepared the ${ }^{13} \mathrm{C}$ form of the protein in order to shift the protein's amide I region away from the absorption region of DHN. As shown in Fig. S6C, three peaks were observed at $1660 \mathrm{~cm}^{-1}, 1681$ $\mathrm{cm}^{-1}$, and $1710 \mathrm{~cm}^{-1}$ when DHN was bound to wild-type $\left[{ }^{13} \mathrm{C}\right] \mathrm{KSI}$. The electric fields corresponding to these peaks are $-136.8 \mathrm{MV} / \mathrm{cm},-93.2 \mathrm{MV} / \mathrm{cm}$, and $-34.1 \mathrm{MV} / \mathrm{cm}$ respectively. Provisionally, we have assigned the $-136.8 \mathrm{MV} / \mathrm{cm}$ peak to a KSI:DHN complex that adopts a productive conformation for catalysis that resembles what we see in $\mathrm{x}$-ray crystallography, because it agrees reasonably well with the calculated field based on the crystal structure $(-127.6 \mathrm{MV} / \mathrm{cm})$. The $-93.2 \mathrm{MV} / \mathrm{cm}$ could correspond to a state in which DHN is not fully H-bonded to the active site, as has been seen in extensive MD simulations carried out by Welborn et al. ${ }^{18}$ We have 
provisionally assigned the '-34.1 MV/cm' peak to carboxylate side-chains on KSI. ${ }^{19}$ We attempted to confirm these assignments with isotope-edited FTIR; unfortunately, as discussed in the SI methods and shown in Fig. S6C, the oxygen atom of the carbonyl in ${ }^{18} \mathrm{O}$-labeled DHN rapidly exchanges with solvent. Without this validation, it remains possible that these provisional assignments are not correct, but since all these derived electric fields are smaller than $-141 \mathrm{MV} / \mathrm{cm}$, we can at least conclude from these IR spectra that DHN experiences a smaller electric field than $19 \mathrm{NT}$ when bound to KSI.

The observation of the multiple populations hints at the possibility of flexibility of the KSI complex in the ground state, where it is able to sample the catalytically active conformation for a fraction of the time. ${ }^{18}$ It is also possible that alternative conformations were present in our original characterization of the KSI:19NT complexes, ${ }^{2}$ but their spectral features were masked by the strong amide signals from the protein.

The possibility of several conformations of the enzyme-substrate complex is consistent with the notion that enzyme-substrate complexes must explore their energy landscapes to locate a catalytically active conformation. ${ }^{20}$ In the present example, this search likely corresponds to subtle motions of the substrate sampling various poses until it finds the TS-like pose (i.e., the -136.8 $\mathrm{MV} / \mathrm{cm}$ feature, which is probably very similar to the pose we captured by crystallography). Whereas this conformation would be sparingly sampled in solution, in the active site, it is stabilized by the preorganized field. In other enzymes, the search for the catalytically active state may take more time because it involves more dramatic conformational changes of the substrate and enzyme (as documented extensively by Richard and co-workers). ${ }^{21,22}$ 


\section{Supplementary Tables}

Table S1 | X-ray diffraction data collection and refinement statistics

\begin{tabular}{|c|c|c|}
\hline & $\begin{array}{c}\text { WT-KSI bound with } \\
\text { dihydronandrolone } \\
\text { (PDB\# 6UFS) }\end{array}$ & $\begin{array}{l}\text { WT-KSI bound with } \\
\text { 19-nortestosterone } \\
\text { (PDB\# 5KP4) }\end{array}$ \\
\hline Resolution range $(\AA)$ & $47.80-1.70$ & $36.29-1.71$ \\
\hline Space group & $\mathrm{P} 2{ }_{1} 2_{1} 2_{1}$ & $\mathrm{P} 2{ }_{1} 2_{1} 2_{1}$ \\
\hline $\mathrm{a}, \AA$ & 35.87 & 33.85 \\
\hline $\mathrm{b}, \AA$ & 73.79 & 72.59 \\
\hline $\mathrm{c}, \AA$ & 95.65 & 94.82 \\
\hline$\alpha,^{\circ}$ & 90.00 & 90.00 \\
\hline$\beta,^{\circ}$ & 90.00 & 90.00 \\
\hline$\gamma,{ }^{\circ}$ & 90.00 & 90.00 \\
\hline No. unique reflections & 33513 & 26203 \\
\hline Completeness, $\%$ & 96.1 & 99.8 \\
\hline Multiplicity & 4.5 & 6.6 \\
\hline$R_{\text {merge }}, \%$ & 9.2 & 9.6 \\
\hline $\mathrm{I} / \sigma_{\text {overall }}$ & 24.6(1.9) & $9.6(2.1)$ \\
\hline \multicolumn{3}{|l|}{ Refinement statistics } \\
\hline No. residues & 256 & 254 \\
\hline No. waters & 267 & 138 \\
\hline Rwork, \% & 18.9 & 20.3 \\
\hline Rfree, \% & 24.9 & 23.9 \\
\hline rmsd bond, $\AA$ & 0.006 & 0.006 \\
\hline rmsd angle, ${ }^{\circ}$ & 0.77 & 0.85 \\
\hline
\end{tabular}


Table S2 | KSI active site electric fields from structural analysis and MD calculations (see Figure S4)

\begin{tabular}{|c|c|c|c|c|c|c|c|c|}
\hline & coordinates & $\begin{array}{l}\text { field } \\
\text { projection }^{a} \\
(\mathrm{MV} / \mathrm{cm})\end{array}$ & $\begin{array}{l}\% \\
\text { aligned }\end{array}$ & $\begin{array}{l}\text { stabilization }^{b} \\
\left(\mathrm{kcal} \mathrm{mol}^{-1}\right)\end{array}$ & $\begin{array}{l}\Delta \text { stabilization } \\
\left(\mathrm{kcal} \mathrm{mol}^{-1}\right)\end{array}$ & $\begin{array}{l}\mathrm{C}=\mathrm{O} \ldots \mathrm{O}_{16} \\
(\AA)\end{array}$ & $\begin{array}{l}\mathrm{C}=\mathrm{O} \ldots \mathrm{O}_{103} \\
(\AA) \\
(\AA)\end{array}$ & $\begin{array}{l}\mathrm{C}=\mathrm{O} \\
\text { angle shift }\end{array}$ \\
\hline \multirow[b]{2}{*}{ DHN } & X-ray (1) & -127.6 & $91 \%$ & -20.8 & 7.2 & 2.67 & 2.73 & reference \\
\hline & $\begin{array}{l}\text { gas-phase } \\
\text { opt. (2) }\end{array}$ & -83.5 & $79 \%$ & -13.6 & reference & 3.18 & 2.79 & $\sim 35^{\circ}$ \\
\hline \multirow[b]{2}{*}{ substrate } & constrained (4) & -112.3 & $88 \%$ & -18.3 & 8.6 & 3.10 & 2.60 & reference \\
\hline & $\begin{array}{l}\text { gas-phase } \\
\text { opt. (3) }\end{array}$ & -59.3 & $70 \%$ & -9.7 & reference & 3.75 & 2.82 & $\sim 31^{\circ}$ \\
\hline \multirow[b]{2}{*}{$19 \mathrm{NT}$} & x-ray (6) & -141.7 & $88 \%$ & -27.9 & 12.9 & 2.57 & 2.65 & reference \\
\hline & $\begin{array}{l}\text { gas-phase } \\
\text { opt. }{ }^{c}(7)\end{array}$ & -76.1 & $75 \%$ & -15.0 & reference & 3.48 & 2.62 & $\sim 55^{\circ}$ \\
\hline \multirow{2}{*}{$\begin{array}{l}\text { inter- } \\
\text { mediate }\end{array}$} & constrained (9) & -144.2 & $96 \%$ & -31.2 & 0.1 & 2.57 & 2.51 & reference \\
\hline & $\begin{array}{l}\text { gas-phase } \\
\text { opt. (8) }\end{array}$ & -144.0 & $92 \%$ & -31.1 & reference & 2.49 & 2.53 & $\sim 8^{\circ}$ \\
\hline $\mathrm{TS}$ & $\begin{array}{l}\text { gas-phase } \\
\text { opt. (5) }\end{array}$ & -144.6 & $95 \%$ & -28.5 & l & 2.70 & 2.48 & / \\
\hline
\end{tabular}

${ }^{a}$ The electric field projection is determined by using the coordinates of the ligand in the KSI active site from x-ray crystallography (or by replacing them with geometry-optimized structures, as explained in text) in short MD simulations.

${ }^{b}$ Stabilization energies are calculated by combining electric field projections and the $\mathrm{C}=\mathrm{O}$ bond dipole magnitude (see Table S5). The bond dipole magnitudes of the substrate and the transition state are used for DHN and 19NT, respectively.

${ }^{c}$ We note that upon superimposing the gas-phase optimized 19NT structure onto the crystal structure, the carbonyl group was translated by $\sim 1.4 \AA$ (Fig. $2 \mathrm{~B}$ ). The big translation was partly due to the artifact of attempting to align the rest of the ligand's multi-ring system. It is very likely that in KSI the positioning of the A ring relative to the oxyanion hole is the main determinant of the ligand's binding mode. Therefore, we only use the alignment against the entire 19NT structure here to qualitatively demonstrate how the observed geometrical perturbation of the carbonyl leads to better alignment and in turn a larger projected field. 
Table S3 | Solvatochromism data of DHN's carbonyl ${ }^{a}$

\begin{tabular}{ccccc}
\hline Solvent & $\begin{array}{c}\text { Peak Position } \\
\bar{v}\left(\mathrm{~cm}^{-1}\right)\end{array}$ & $\begin{array}{c}\text { FWHM } \\
\left(\mathrm{cm}^{-1}\right)\end{array}$ & $\begin{array}{c}\text { Electric Field Mean } \\
\langle|\vec{F}|>(\mathrm{MV} / \mathrm{cm})\end{array}$ & $\begin{array}{c}\text { Electric Field Standard } \\
\text { Deviation }(\mathrm{MV} / \mathrm{cm})\end{array}$ \\
\hline Hexane & 1726.2 & 12.9 & $-0.01 \pm 0.03$ & 0.78 \\
Dibutylether & 1722.4 & 13.6 & $-8.85 \pm 0.77$ & 5.4 \\
Tetrahydrofuran & 1717.5 & 13.2 & $-19.6 \pm 0.35$ & 7.8 \\
Acetonitrile & 1712.4 & 14.5 & $-29.9 \pm 0.86$ & 12.9 \\
Dichloromethane & 1711.6 & 12.5 & $-31.2 \pm 0.64$ & 17.0 \\
Chloroform & 1709.4 & 18.2 & $-33.7 \pm 1.2$ & 15.1 \\
Methyl sulfoxide & 1709.3 & 15.9 & $-35.3 \pm 0.45$ & 10.9 \\
Water & 1690.5 & 25.1 & $-74.6 \pm 0.58$ & 30.0 \\
\hline
\end{tabular}

${ }^{a}$ See Fig. 4 in the main text

Table S4 | Electric fields of ligands bound to KSI as determined by Vibrational Stark Effects

\begin{tabular}{|c|c|c|c|c|c|c|}
\hline \multirow{3}{*}{$\begin{array}{c}\text { Solvation } \\
\text { environment } \\
\text { Peak Position } \bar{v} \\
\left(\mathrm{~cm}^{-1}\right)\end{array}$} & \multicolumn{3}{|c|}{$\begin{array}{c}\text { DHN }\left(\mathrm{C}={ }^{16} \mathrm{O}\right) \\
\text { model for substrate }\end{array}$} & \multicolumn{3}{|c|}{$\begin{array}{l}19 \mathrm{NT}\left(\mathrm{C}={ }^{16} \mathrm{O}\right) \\
\text { model for transition state }\end{array}$} \\
\hline & \multicolumn{2}{|c|}{ KSI } & \multirow{2}{*}{1690.5} & \multirow{2}{*}{ KSI } & \multirow{2}{*}{$\mathrm{D}_{2} \mathrm{O}$} & \multirow{2}{*}{$\begin{array}{l}\mathrm{D}_{2} \mathrm{O} \text { (in DHN's } \\
\text { solvent sphere }{ }^{d} \text { ) }\end{array}$} \\
\hline & $1660.3^{a}$ & $1681.4^{a}$ & & & & \\
\hline FWHM $\left(\mathrm{cm}^{-1}\right)$ & 9.0 & 10.0 & 25.1 & 3.8 & 40.6 & I \\
\hline $\begin{array}{l}\text { field projection } \\
\qquad(\mathrm{MV} / \mathrm{cm})^{b}\end{array}$ & -136.8 & -93.2 & -74.6 & -141.3 & -80.4 & $\begin{array}{c}-74.6 \\
\cos \left(31^{\circ}\right)\end{array}$ \\
\hline $\begin{array}{l}\text { Stabilization }^{c} \\
\left(\mathrm{kcal} \mathrm{mol}^{-1}\right)\end{array}$ & -22.3 & -15.2 & -12.2 & -27.8 & -15.8 & -12.6 \\
\hline \multirow{2}{*}{\multicolumn{7}{|c|}{$\begin{array}{l}{ }^{a} \text { Both IR peaks from VSE spectroscopy are provisionally assigned to DHN - see Fig. 3C and Fig. S5. } \\
\text { The electric field projection as determined from VSE spectroscopy (and using a solvent-based calibration curve) } \\
\text { Stabilization energies are calculated by combining electric field projections and the C=O bond dipole magnitude (see }\end{array}$}} \\
\hline & & & & & & \\
\hline \multicolumn{7}{|c|}{$\begin{array}{l}\text { Table S5) }{ }^{d} \text {. In rapid chemical reactions that proceed in solvent, the TS would form initially in the solvent sphere that } \\
\text { was equilibrated with the substrate. Since the carbonyl reorients by } 31^{\circ} \text { in } \mathrm{TS} \text {, the solvent field would be out of } \\
\text { alignment with the } \mathrm{C}=\mathrm{O} \text { dipole, and the projected field would be lower by a factor of } \cos \left(31^{\circ}\right) \text {. }\end{array}$} \\
\hline
\end{tabular}


Table S5 | Optimal geometries of ligand structures from DFT

\begin{tabular}{ccccc}
\hline & O charge (a.u) & C charge (a.u) & C-O (̊) & C-O dipole (D) \\
\hline Substrate & -0.550 & 0.627 & 1.21 & 3.4 \\
Transition State & -0.717 & 0.663 & 1.24 & 4.1 \\
Intermediate & -0.802 & 0.685 & 1.26 & 4.5 \\
\hline
\end{tabular}




\section{Supplementary Figures}

A

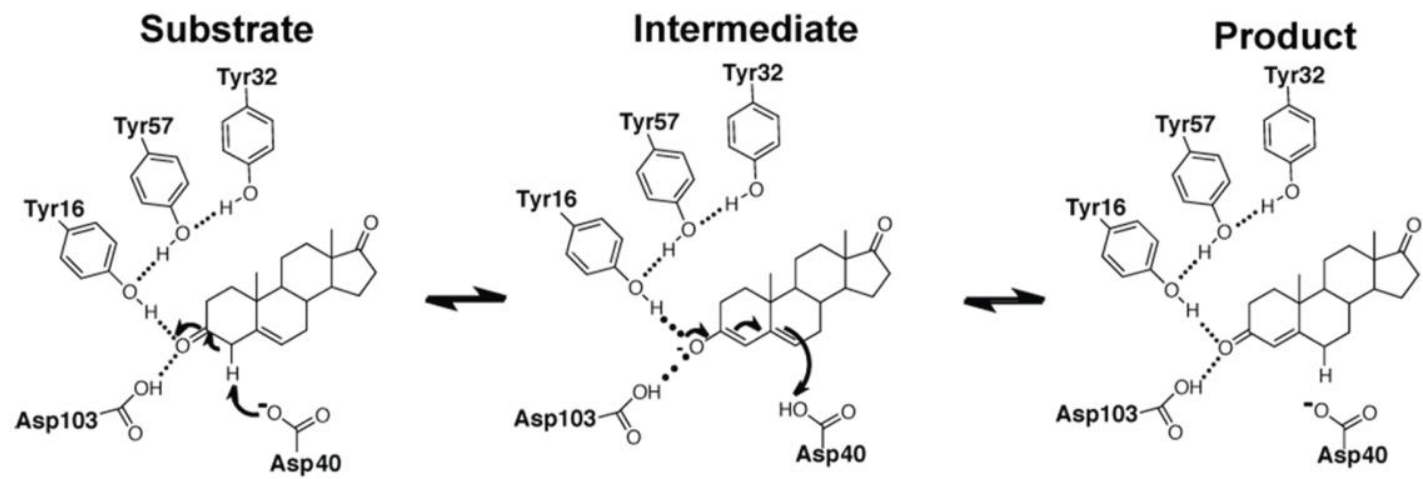

B

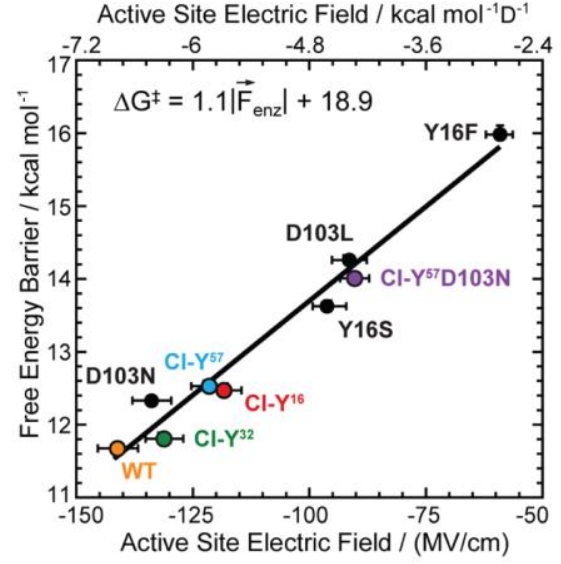

Figure S1 | Linear correlation between KSI's electric field and rate acceleration

(A) An extended H-bond network composed of the tyrosine triad $\left(\mathrm{Tyr}^{16}, \mathrm{Tyr}^{57}\right.$ and $\mathrm{Tyr}^{32}$ ) and Asp ${ }^{103}$ interacts with the carbonyl group of the substrate, stabilizing the accumulated negative charge in the TS/Intermediate state. ${ }^{23}$ (B) The H-bond interactions enables KSI to exert a large electric field on the carbonyl. The magnitude of the electric field is linearly correlated with the free energy barrier of the catalyzed reaction in wild-type and mutants with canonical and noncanonical amino acid substitutions ( $\Delta G^{\ddagger}$ was obtained from $k_{\text {cat }}$ using transition state theory). From the slope of the linear correlation and fitting to Eq. 2, $\left(\left|\vec{\mu}_{T S}\right|-\left|\vec{\mu}_{R}\right|\right)$ was estimated to be 1.1D. By extrapolating to zero field, the electrostatic contribution to $\Delta \Delta G^{\ddagger}$ was estimated to be $7.3 \mathrm{kcal} \mathrm{mol}^{-}$ ${ }^{1}$, contributing $70 \%$ of KSI's total rate acceleration. ${ }^{2,12}$ 

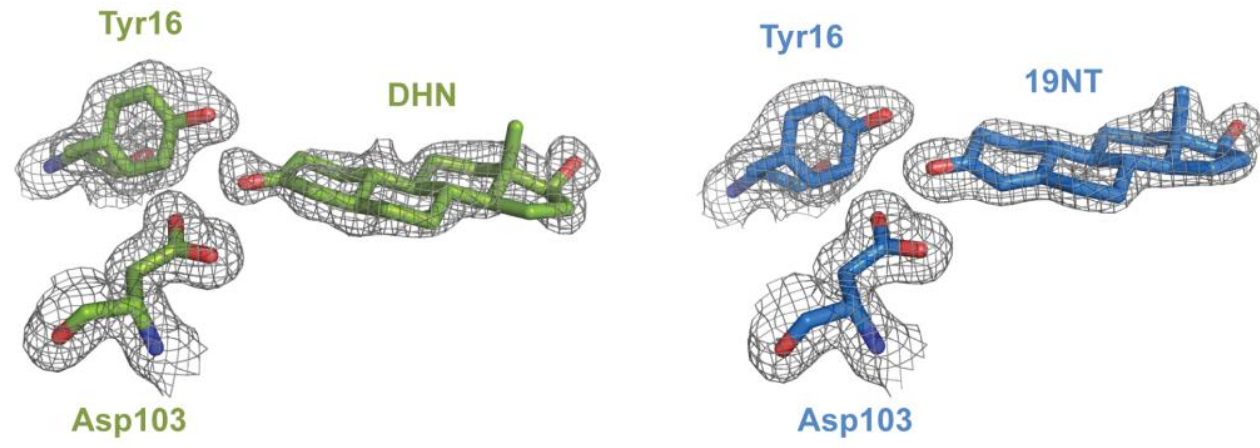

Figure S2 | Electron density maps of the oxyanion hole residues and the bound ligand showing their relative positioning

The active site of wild type KSI bound to DHN (green) and wild-type KSI bound to 19NT (blue). In both structures, the electron density around the carbonyl bond is sufficient to support a TS-like geometry modeled from the gas phase calculations. 

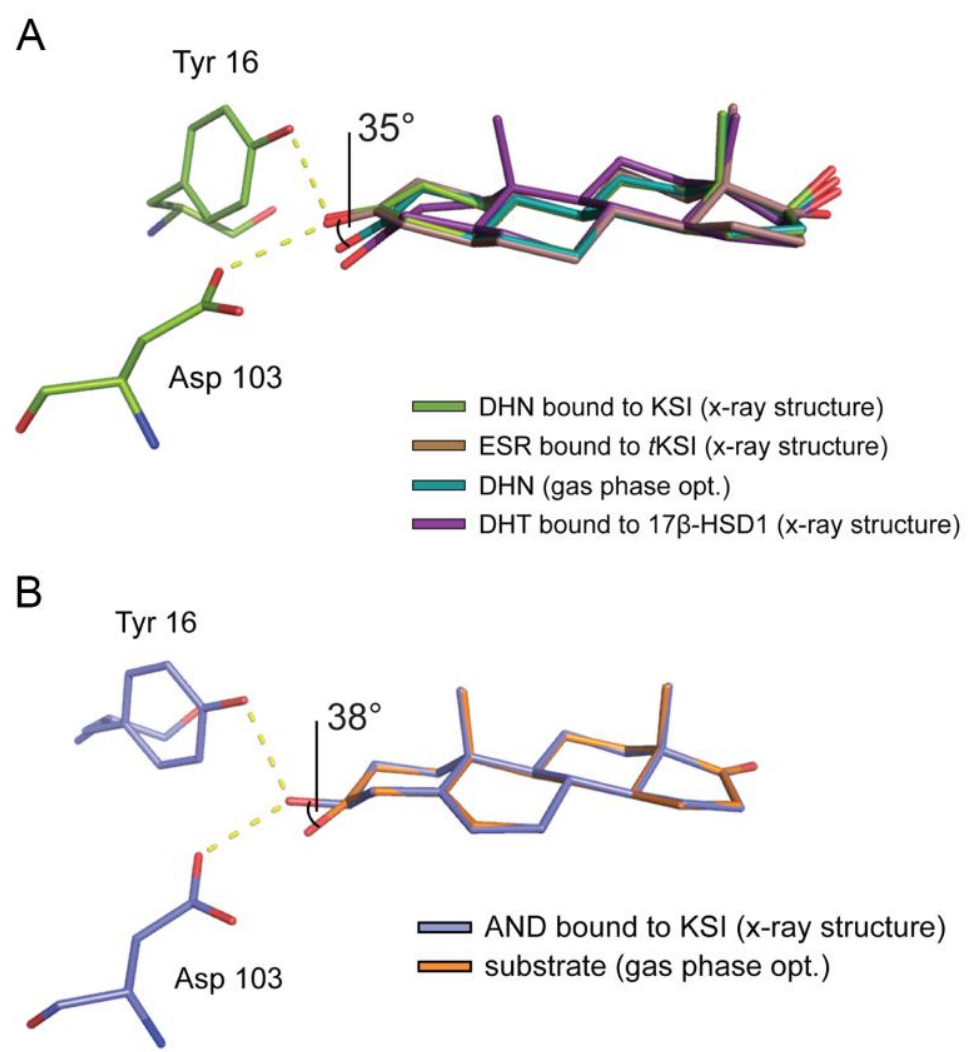

Figure S3 | Geometrical change between steroids bound to enzymes and in the gas phase.

(A) The perturbed 'TS-like' geometry for DHN bound to KSI is recapitulated by a different steroid, $5 \alpha$-estran-3,7-dione (ESR), bound to a different KSI orthologue, $t$ KSI, but absent in dihydrotestosterone (DHT) bound to 17 $\beta$-hydroxysteroid dehydrogenase, which more closely resembles the gas-phase optimized geometry of DHN. (B) The gas-phase optimized substrate, 5androstenedione, is compared with substrate analog, androstane-3beta-ol-17-one (AND), bound to the catalytically inactive $p \mathrm{KSI}^{\mathrm{D} 40 \mathrm{~N}}$ (PDB 1E3R). The structural difference at $\mathrm{C}_{3}$ between the substrate and AND (i.e. $\mathrm{sp}^{2}$ hybridization to $\mathrm{sp}^{3}$ hybridization) mimics the angle change at the carbonyl upon the formation of the TS/Intermediate (Fig. 1): AND's C-O bond in shifted from the substrate by $38^{\circ}$, inherently aligned with KSI's electric field $\left(\sim 31^{\circ}\right)$. The carbonyl of DHN is distorted by the field to achieve the same optimal interaction. 


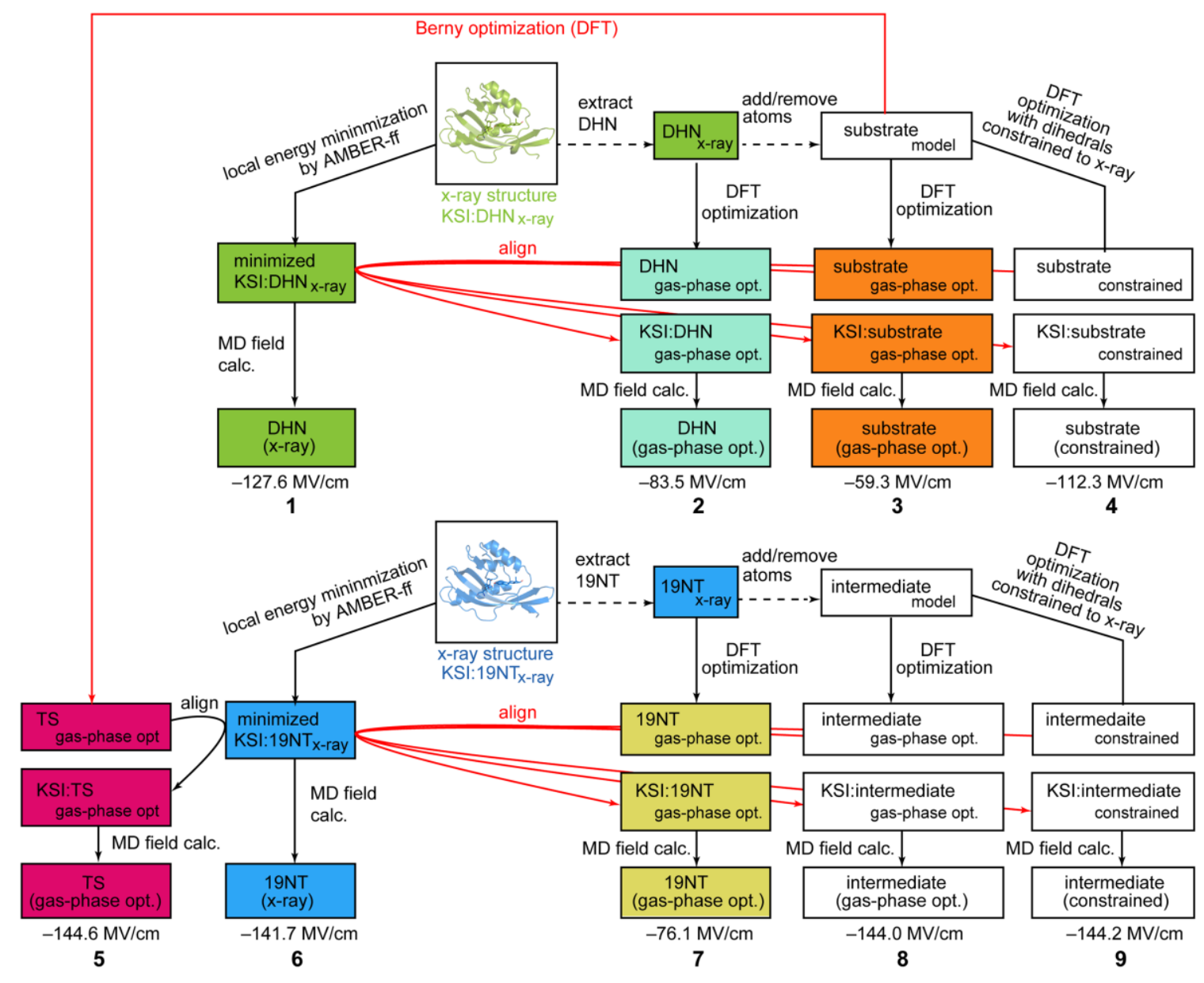

Figure S4 | Modelling scheme of different KSI:ligand complexes for MD calculations.

Each construct is given a number whose corresponding simulation results are listed in Table S2. The modelling strategy is also explained and examined in SI Methods and in supplementary discussion S1. 


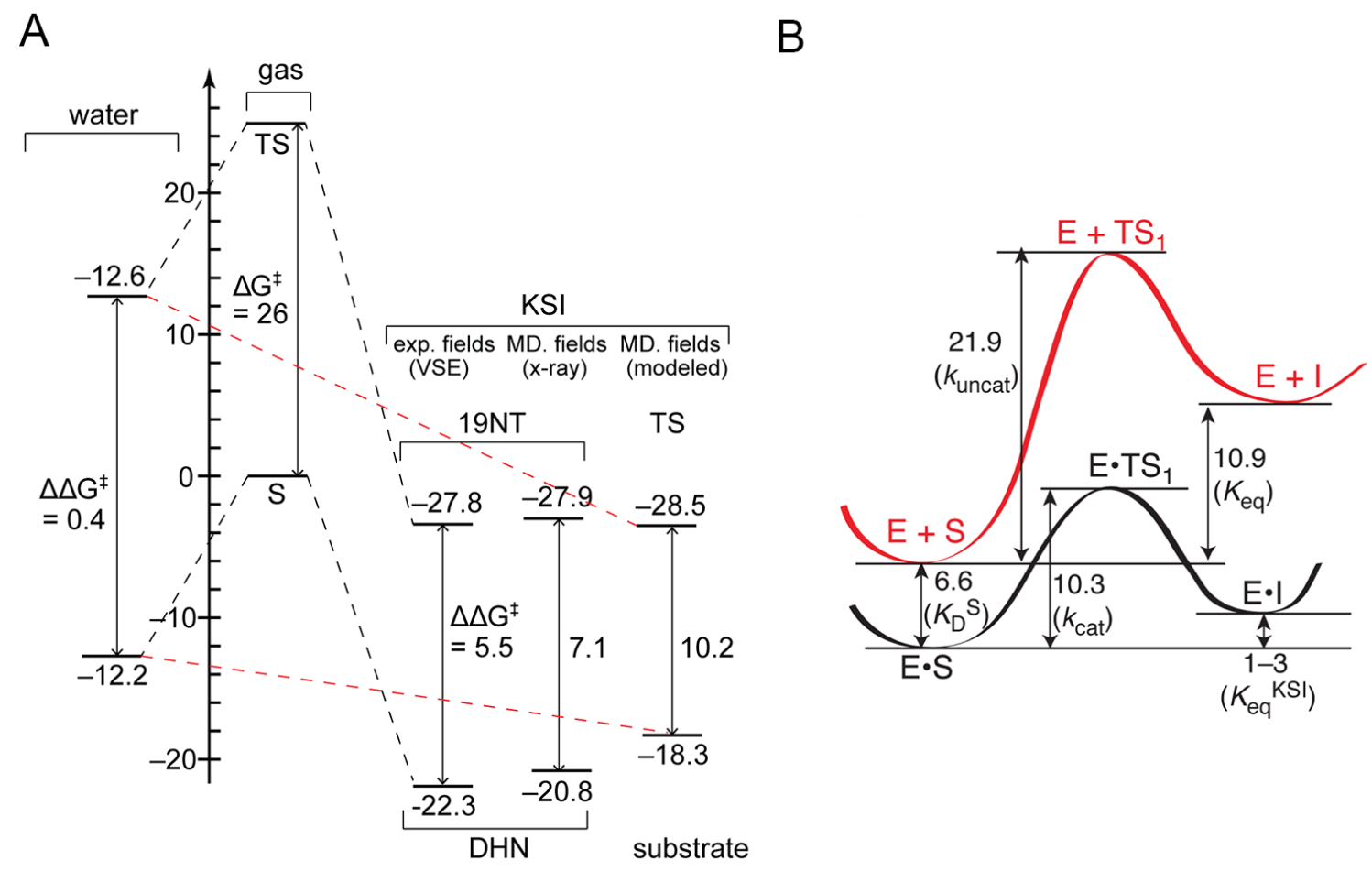

\section{Figure S5 | Stabilization energies in different environments and the free energy profiles of the isomerization reaction in KSI and in aqueous solution}

(A) The relative free energy of the substrate and the TS are estimated in gas phase, water and KSI. The stabilization energy of the TS in water is estimated under the assumption that that the solvent reaction field is equilibrated with the reactant's dipole orientation (which is $31^{\circ}$ out of alignment with the TS, Fig. 1B), whereas the stabilization energy of the substrate in water assumes $100 \%$ alignment between the solvent reaction field and the reactant dipole (Table S4). The stabilization energy in KSI is estimated in three ways: (1) from the measured electric field projections on the carbonyl of 19NT and DHN (Table S4) by VSE, which is likely an underestimation of KSI's preferential stabilization on the TS over the substrate because the actual substrate, 5-androstenedione, would not be as easily distorted to TS-structure as DHN is (Figure 2B); (2) from the MD calculated electric fields based on the actual crystal structures of the KSI:19NT and KSI:DHN complexes (Table S2); (3) from the MD calculated electric fields based on the modeled structures of the KSI:substrate and KSI:TS complexes (Table S2 in bold), which is likely an overestimation of the preferential stabilization energy because the alignment of the substrate into the DHN crystal structure coordinate is not ideal. Red dashed lines indicate the additional stabilization energies provided by the enzyme environment over solutions upon ligand binding $\left(6.1 \mathrm{kcal} \mathrm{mol}^{-1}\right.$ for substrate; $15.9 \mathrm{kcal} \mathrm{mol}^{-1}$ for transition state). (B) Reaction coordinate diagram for the enolization of 5-androstenedione (S) to the dienolate intermediate (I), either in KSI (black) or in solution (red). Numbers correspond to free energy differences in $\mathrm{kcal} \mathrm{mol}^{-1}$. Black arrows correspond to measured quantities and are associated with the binding/rate constants in parenthesis. ${ }^{14-16,24-26}$ The figure is adapted from Fried, S.D. Ph.D. Dissertation, Stanford University, 2014. 
A

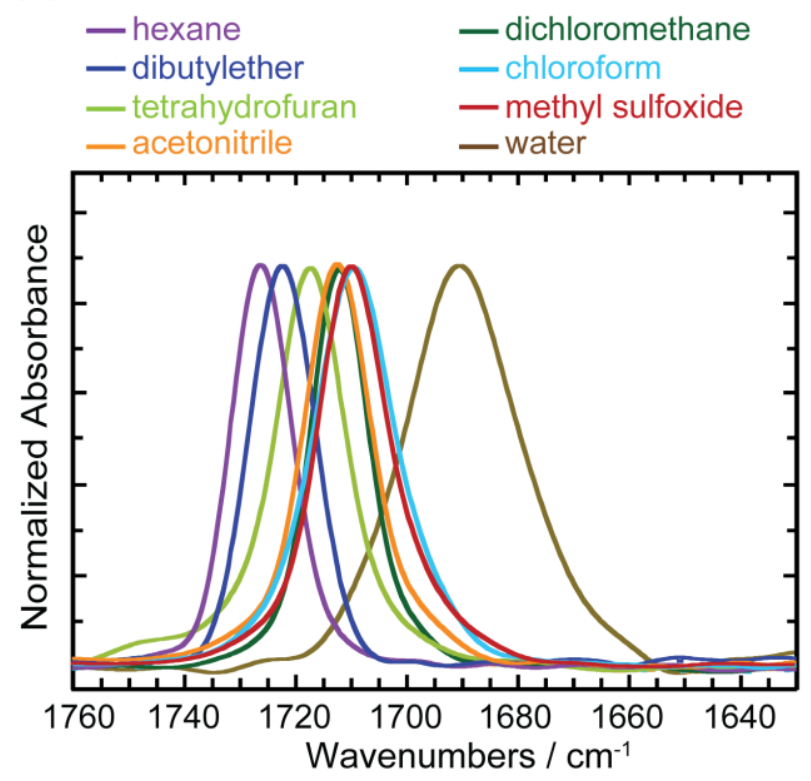

B

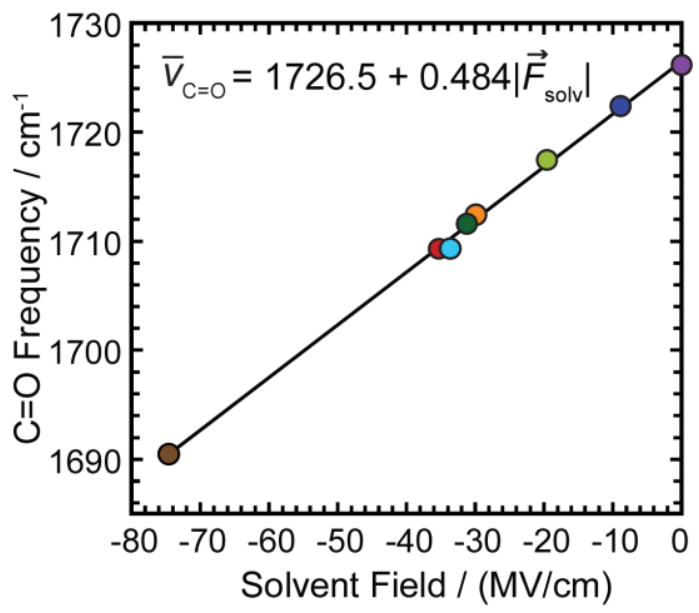

C — sample (DHN-19NT) …...... $\left.{ }^{12} \mathrm{C} \mathrm{KSI} \mathrm{background} \mathrm{(redshift} 42 \mathrm{~cm}^{-1}\right)$ ---- blank (DHN-DHN) -.-- absolute absorbance (KSI:DHN)

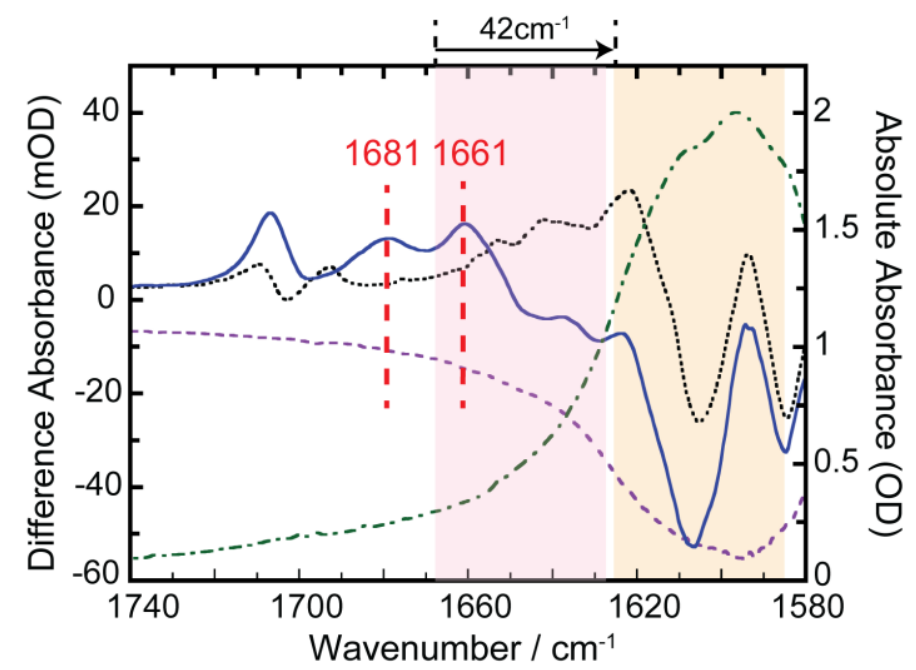

\section{Figure S6 | Infrared spectra of DHN in different solvents and bound to wild type $\left[{ }^{13} \mathrm{C}\right] \mathrm{KSI}$}

(A) Vibrational solvatochromism for DHN's carbonyl in solvents with increasing polarity. (B) The average value of the solvent fields exerted onto the $\mathrm{C}=\mathrm{O}$ of $\mathrm{DHN}$ display a linear correlation with the $\mathrm{C}=\mathrm{O}$ stretching frequency $\left(R^{2}=0.99\right)$. (C) The frequency range of the carbonyl peak (s) of DHN is determined to be between $1691 \mathrm{~cm}^{-1}$ and $1658 \mathrm{~cm}^{-1}$, because the DHN bound to WT KSI is expected to experience an electric field larger than that in water and smaller than or similar to that experienced by TS-like ligand 19NT. Because the amide I band of ${ }^{12} \mathrm{C}$ KSI dominates the signal in this frequency range (light pink shade), we employed ${ }^{13} \mathrm{C}$-labeling to redshift the amide I band by $42 \mathrm{~cm}^{-1}$ from $\sim 1642 \mathrm{~cm}^{-1}$ to $\sim 1600 \mathrm{~cm}^{-1}$ (light yellow shade), 
leaving a relatively flat baseline in the region of interest ('KSI background'). Two peaks at $1660.3 \mathrm{~cm}^{-1}$ (FWHW $\sim 9 \mathrm{~cm}^{-1}$, intensity $\sim 6 \mathrm{mOD}$ ) and $1681.4 \mathrm{~cm}^{-1}$ (FWHW $\sim 10 \mathrm{~cm}^{-1}$, intensity $\sim 4 \mathrm{mOD}$ ) were consistently observed above the background and were provisionally assigned as the $\mathrm{C}={ }^{16} \mathrm{O}$ stretch of DHN sulfate bound in the active site. The peak at $\sim 1710 \mathrm{~cm}^{-1}$ likely comes from the carboxyl group of amino acid side chains. ${ }^{19}$ Comparing the absorption spectrum of the KSI complex (green, right y axis) with the difference absorption spectrum (blue, left y axis), we see that the total absorbance from the enzyme complex is about 25 times more intense than the absorbance from the ligand's carbonyl.

As discussed in the experimental section, we also attempted to confirm the identities of the two peaks observed with isotope labeled substrate. $\left[{ }^{16} \mathrm{O}\right] \mathrm{DHN}$ sulfate was used as the sample and $\left[{ }^{18} \mathrm{O}\right] \mathrm{DHN}$ sulfate was used as the reference. The isotope labeling on the carbonyl would ensure an otherwise identical IR background except a positive feature from $\mathrm{C}={ }^{16} \mathrm{O}$ vibration and a negative feature from $\mathrm{C}={ }^{18} \mathrm{O}$ vibration that are separated by approximately $30 \mathrm{~cm}^{-1}$. In reality, however, the measurements were rendered unreliable by two factors: 1) The steep slope from $1600 \mathrm{~cm}^{-1}$ to $1640 \mathrm{~cm}^{-1}$ in the difference absorbance spectrum due to the ${ }^{13} \mathrm{C}$-labeled amide signal obscured the $\mathrm{C}={ }^{18} \mathrm{O}$ stretch of DHN sulfate; and 2) the isotope labeled carbonyl of DHN hydrolyzes back to $\mathrm{C}={ }^{16} \mathrm{O}$ quickly with the presence of trace $\left[{ }^{16} \mathrm{O}\right]$ over the course of IR sample preparation and measurements, further decreasing the IR signals. 


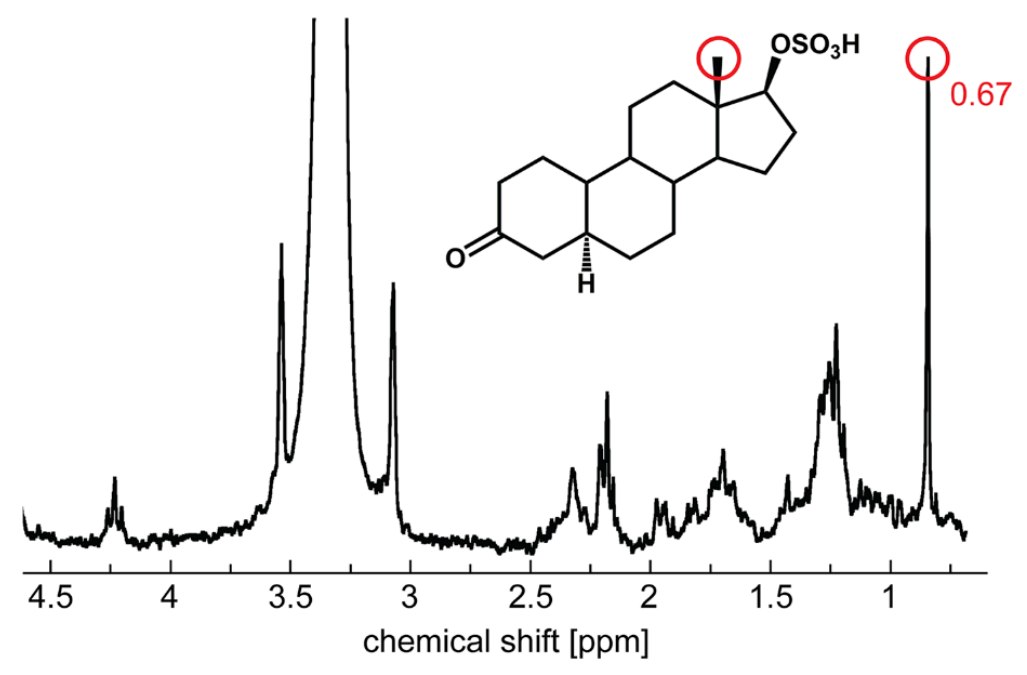

\section{Figure S7 | ${ }^{1}$ H-NMR spectrum of purified dihydronandrolone sulfate}

The signal corresponds at $0.67 \mathrm{ppm}$ corresponds to the three methyl group protons circled. This was used to calibrate the concentration of DHN and DHN sulfate against 19NT and trimethylsilylpropanoic acid (TSP- $\mathrm{d}_{4}$ ) standard. The big peak around 3.3ppm comes from the trace $\mathrm{H}_{2} \mathrm{O}$ in deuterated solvent dimethyl sulfoxide- $\mathrm{d}_{6}{ }^{27}$ 


\section{References}

(1) Wu, Y.; Fried, S. D.; Boxer, S. G. Biochemistry 2015, 54, 7110-7119.

(2) Fried, S. D.; Bagchi, S.; Boxer, S. G. Science 2014, 346, 1510-1513.

(3) Oltrogge, L. M.; Boxer, S. G. ACS Cent. Sci. 2015, 1, 148-156.

(4) Wilson, I. A.; Stura, E. A. J. Cryst. Growth 1991, 110, 270-282.

(5) Kabsch, W. Acta Crystallogr. Sect. D Biol. Crystallogr. 2010, 66, 133-144.

(6) Otwinowski, Z.; Minor, W. Methods Enzymol. 1997, 276, 307-326.

(7) Gonzalez, A.; Tsai, Y. http://smb.slac.stanford.edu/facilities/software/xds/\#autoxds_script 2010.

(8) McCoy, A. J.; Grosse-Kunstleve, R. W.; Storoni, L. C.; Read, R. J. Acta Cryst. 2005, D61, 458464.

(9) Adams, P. D.; Grosse-Kunsleve, R. W.; Hung, L.-W.; Loerger, T. R.; McCoy, A. J.; Moriarty, N. W.; Read, R. J.; Sacchettini, J. C.; Sauter, N. K.; Terwilliger, T. C. Acta Cryst. 2002, D58, 19491954.

(10) Emsley, P.; Cowtan, K. Acta Cryst. 2004, D60, 2126-2132.

(11) DeLano, W. L. http://www.pymol.org.

(12) Wu, Y.; Boxer, S. G. J. Am. Chem. Soc. 2016, 138, 11890-11895.

(13) Fried, S. D.; Bagchi, S.; Boxer, S. G. J. Am. Chem. Soc. 2013, 135, 11181-11192.

(14) Schwans, J. P.; Kraut, D. A.; Herschlag, D. Proc. Natl. Acad. Sci. U. S. A. 2009, 106, 14271-14275.

(15) Hawkinson, D. C.; Eames, T. C. M.; Pollack, R. M.; Hawkinson, D. C.; Eames, T. C. M.; Pollack, R. M. Biochemistry 1991, 30, 10849-10858.

(16) Zeng, B.; Pollack, R. M. J. Am. Chem. Soc. 1991, 113, 3838-3842.

(17) Schneider, S. H.; Boxer, S. G. J. Phys. Chem. B 2016, 120, 9672-9684.

(18) Welborn, V. V.; Head-gordon, T. J. Am. Chem. Soc. 2019, 141, 12487-12492.

(19) Barth, A. Prog. Biophys. Mol. Biol. 2000, 74, 141-173.

(20) Hammes-Schiffer, S. Biochemistry 2013, 52, 2012-2020.

(21) Richard, J. P. J. Am. Chem. Soc. 2019, 141, 3320-3331.

(22) Amyes, T. L.; Richard, J. P. Biochemistry 2013, 52, 2021-2035.

(23) Fafarman, A. T.; Sigala, P. A.; Schwans, J. P.; Fenn, T. D.; Herschlag, D.; Boxer, S. G. Proc. Natl. Acad. Sci. U. S. A. 2012, 109, E299-308.

(24) Hawkinson, D. C.; Pollack, R. M.; Ambulos, N. P. Biochemistry 1994, 33, 12172-12183.

(25) Pollack, R. M. Bioorg. Chem. 2004, 32, 341-353.

(26) Fried, S. D.; Boxer, S. G. Proc. Natl. Acad. Sci. U. S. A. 2013, 110, 12271-12276.

(27) Fulmer, G. R.; Miller, A. J. M.; Sherden, N. H.; Gottlieb, H. E.; Nudelman, A.; Stoltz, B. M.; Bercaw, J. E.; Goldberg, K. I. Organometallics 2010, 29, 2176-2179. 
